\title{
Spin Density Distribution and Interaction Mechanisms in Thiazyl-based Magnets
}

\author{
J. Campo ${ }^{1}$ J. Luzón ${ }^{1}$, F. Palacio ${ }^{1}$, and J. Rawson ${ }^{2}$ \\ ${ }^{I}$ Instituto de Ciencia de Materiales de Aragon, CSIC-Universidad de Zaragoza, \\ E-50009, Zaragoza, Spain \\ ${ }^{2}$ Department of Chemistry, The University of Cambridge, Lensfield Road, Cambridge \\ CB2 1EW, UK
}

\section{Introduction}

This chapter presents a study of the magnetic properties and magnetic interaction mechanisms in a series of thiazyl radicals, which are of particular interest in the field of molecular magnetism.

The chapter is organized as follows. First, there is an introduction to organic molecular magnetism and thiazyl radicals. The importance of an accurate knowledge of the spin density distribution in order to understand the mechanisms of the magnetic interactions is discussed. This is highlighted with complimentary experimental and theoretical $a b$ initio determinations of the spin density distribution in the dithiadiazolyl radical, $p-\mathrm{O}_{2} \mathrm{NC}_{6} \mathrm{~F}_{4} \mathrm{CNSSN}^{*}$. An ab-initio computation of the magnetic interactions in this radical is presented in relation to its spin density distribution and magnetic behaviour. This approach is then extended to other dithiadiazolyl and thiazyl radicals. Finally, the conclusions revealed from this study are summarized.

\subsection{Organic Magnets}

Since the discovery of the $\square$-phase of $p$-nitrophenyl nitronyl nitroxide ( $p$-NPNN) as the first organic ferromagnet free of metallic ions [1] the design of new purely organic 
ferromagnets has been the subject of a considerable amount of research.

The presence of unpaired electrons are the basis of magnetic phenomena. In the case of organic molecules they can be obtained in two different ways. One is based on charge-transfer processes between electron donor and electron acceptor molecules to produce open-shell cations and anions. TDAE- $\mathrm{C}_{60}$ [tetrakis (dimethylamino) ethylene$\mathrm{C}_{60}$ ], which exhibits long-range magnetic ordering at $16.1 \mathrm{~K}$, is the best representative example of this approach [2].

The second approach, which is most commonly followed, is the generation of openshell molecules, the so-called free-radicals. In the design of free-radical-based magnets there are several difficulties that have to be overcome.

Almost all the free radicals are unstable species due to their preference of organic systems to form closed-shell configurations via oxidation, reduction or covalent bond formation. Several strategies are used to increase the kinetic stability of the free radicals. Steric shielding of the atoms containing the unpaired electrons is often employed to inhibit covalent bond formation via a free radical mechanism. A second strategy is the use of Coulombic electrostatic repulsions for keeping apart the reactive centres. The tendency of organic systems to form closed-shell configurations via bond formation is typically reflected in a propensity to dimerize in the solid state, which renders them diamagnetic. In order to provide thermodynamic stabilisation of the radical centre it is desireable to provide a singly occupied molecular orbital which is low-lying. This is typically achieved through the introduction of electronegative heteroatoms and/or $\square$-delocalization of the unpaired electrons and decreases its reactivity. The reader interested in a more extended revision of the strategies for stabilizing free radicals is referred to ref. [3].

The interaction between unpaired electrons from different radicals is a necessary requirement for obtaining long-range magnetic order in the solid. Therefore, these strategies for stabilizing free radicals based on the isolation of the unpaired electrons must be employed in moderation. In other words, the kinetic stability and the strength of the magnetic interaction of the free radicals are two opposing requirements that must be balanced in the design of purely organic systems that show bulk magnetic behaviour. A consequence of this balance is the low ordering temperatures of these kinds of materials, almost all of them quite below liquid helium temperature $(4.2 \mathrm{~K})$, which greatly limits the applications of such systems.

In organic molecular systems the two main sources of magnetic anisotropy are magnetic dipolar interactions and spin-orbit coupling. Both sources usually have a small magnitude and purely organic magnets are considered as isotropic Heisenberg systems. The weakness of the dipolar interactions is due to the delocalization of the unpaired electrons. On the other hand, the unpaired electrons are located in molecular orbitals composed of $p$ atomic orbitals with no orbital moment. Therefore, the spin-orbit coupling anisotropy comes from the spin-orbit coupling of the ground state with excited states with orbital angular momentum, and is a second-order term. This low magnetic anisotropy introduces another difficulty in the design of purely organic magnets, since in Heisenberg systems long-range magnetic ordering is only possible if the magnetic interactions are propagated in a three-dimensional network. 
In summary, there are only three requirements for obtaining long-range magnetic ordering in purely organic solids: the existence of magnetic centres, the magnetic interaction among these magnetic centres and the propagation of these magnetic interactions throughout the solid. In practice it proves extremely difficult to achieve all these goals in any one single system. In particular, the last two requirements imply a crucial role of the unpaired electron distribution in the free radicals and of the packing of these free radicals in the crystal.

Among the systems showing long-range magnetic order, those which exhibit a spontaneous magnetization are of particular interest, since they can potentially lead to applications, e.g. magnetic sensors and magneto-optic applications. The different approaches for the design of materials with spontaneous magnetization are described in the next section.

\subsection{Strategies for Obtaining Spontaneous Magnetization}

As mentioned above, a desired property of the molecular magnetic materials is a long-range spontaneous magnetization. Several strategies have been proposed in order to achieve this spontaneous magnetization.

\subsubsection{Orthogonal Orbitals}

In the Anderson formalism for the magnetic exchange interaction the kinetic term is usually dominant and the magnetic interaction is antiferromagnetic. However, when the net overlap between the natural magnetic orbitals is zero, i.e. the orbitals are orthogonal, the kinetic term vanishes and the magnetic interaction is ferromagnetic. This mechanism, for instance, has been used in order to explain the ferromagnetism below $90 \mathrm{~K}$ of the Prussian-blue structured $\mathrm{CsNi}^{\mathrm{II}}\left[\mathrm{Cr}^{\mathrm{III}}(\mathrm{CN})_{6}\right] \cdot 2 \mathrm{H}_{2} \mathrm{O}[4]$.

\subsubsection{Spin Polarization (McConnell I Mechanism)}

Within the Unrestricted Hartree-Fock (UHF) formalism, the spin polarization phenomenon arises from the different exchange interaction between the unpaired electron in the singly occupied molecular orbital (SOMO) with the 'spin up' ( $\square$ ) and 'spin down' ( $\square$ ) electrons in the fully occupied molecular orbitals (FOMO). In the UHF formalism the electron-electron repulsion between the unpaired electron of $\square$ spin of the SOMO with $\square$-spins in the FOMOs will be less than the repulsion between the SOMO $\square$-spin and the corresponding $\square$-spins in the FOMOs. In order to minimise this electronelectron repulsion the FOMOs' $\square$-spins try and adopt regions of space not occupied by the SOMO $\square$-spin. As a consequence the $\square$ and $\square$ spins have slightly different spatial distributions. The total spin density distribution is the difference between the total $\square$ and $\square$-spin distributions and often leads to negative spin density accumulating in regions where the SOMO $\square$-spin has no contribution. The correlation among the electrons, not considered in the Hartree-Fock formalism, also influences the spin polarization effect.

The basis of the MacConnell I mechanism is that when a region of negative spin density in one molecule overlaps with a region of positive spin density in another 
molecule, a ferromagnetic coupling should be expected. In order to support this mechanism McConnell proposed a new Hamiltonian version for the magnetic interaction between two molecules, A and B [5]

$$
H=\square 2 \vec{S}_{A} \vec{S}_{B} \bigsqcup_{i j} J_{i j} \square_{A}^{i} \square_{B}^{j}
$$

Here $\boldsymbol{S}_{A}$ and $\boldsymbol{S}_{B}$ are the total spins on A and B. $J_{i j}$ is a magnetic coupling constant between the $i$ and $j$ atoms located in the A and B molecules respectively. $\square_{\mathrm{A}}^{\mathrm{i}}$ and $\square_{\mathrm{B}}^{\mathrm{j}}$ are the spin populations on the $i$ and $j$ atoms. Since the magnetic coupling constants are usually negative, a ferromagnetic interaction between the two molecules can be achieved if the spin densities of the interacting atoms have opposite signs.

The above expression has been shown to lack theoretical rigour and cannot be naively applied by considering only the closest atoms with high spin populations between the two molecules [5,6]. Whilst it is frequently invoked to explain the magnetic exchange between free radicals, it should be applied with some caution.

\subsubsection{McConnell II Mechanism}

This mechanism was proposed for charge-transfer salts composed of chains of alternating electron-donor and electron-acceptor molecules in which the SOMO orbitals of the donor in the neutral state are degenerate [7]. The triplet state of the neutral donor, according to the Hund's rule for degenerate states, induces a triplet state in the chargetransfer state through a configuration interaction. Such a triplet state is propagated along the donor-acceptor chain. Several modifications of this mechanism have been proposed, where the triplet state of the charge-transfer state is stabilized through configuration interaction with different excited triplet states [8, 9, 10].

The McConnell II mechanism was proposed to explain the bulk ferromagnetic behaviour of the $\left[\mathrm{Fe}\left(\mathrm{Cp}_{2}{ }^{*}\right)\right]^{+}[\mathrm{TCNE}]^{-}$compound below $4.8 \mathrm{~K}$ [11]. However, a polarized neutron diffraction experiment suggests the McConnell I mechanism may be more adequate to explain the ferromagnetic interaction between the donor and the acceptor molecules [12].

\subsubsection{Ferrimagnetism}

Up to now, all the above mechanisms aiming to design materials with long-range spontaneous magnetization are based on the achievement of ferromagnetic interactions. On the other hand, the last two strategies profit from the tendency of magnetic interaction to be antiferromagnetic. The so-called "ferrimagnetic approach" is based in the construction of chains containing alternating magnetic centres with unequal spin values. This is the strategy used in the bimetallic approach for inorganic molecular magnets. Although a similar strategy has been proposed for organic magnets, it is only very recently that the first example has been discovered [13].

\subsubsection{Weak Ferromagnetism}

There are compounds whose magnetic behaviour at high temperature can be ascribed to an antiferromagnetic material but which show a weak spontaneous magnetization 
below a transition temperature. This phenomenon, known as weak ferromagnetism, was explained by Dzyaloshinskii [14] and Moriya [15] as an antiferromagnetic structure in which there exists a canting angle between the magnetic moments of different sub-lattices with opposite signs of the magnetic moments. This non-collinearity of the magnetic moments results in a net magnetization.

There are two main mechanisms that can produce the non-colinearity of the magnetic moments. In the first one, the non-colinearity of the magnetic moments reflects the non-colinearity of the direction of the local magnetic anisotropy. The second mechanism is due to the combination of the spin-orbit coupling and the magnetic interaction between two magnetic centres. This mechanism can be accounted by a new term in the magnetic interaction Hamiltonian:

$$
H_{D M}=\square \vec{D} \cdot\left(\vec{S}_{A} \square \vec{S}_{B}\right)
$$

The conditions allowing canting between two magnetic moments are very strict. In relation to the crystal field, the nuclear and the magnetic unit cells must be the same and the sites whose magnetic moments are non-colinear must not be related by an inversion centre. In other words, weak ferromagnetism is favoured in molecular magnetism due to the higher propensity for low-symmetry molecules to adopt structures without inversion centre. The organic magnets with the highest transition temperatures are weak ferromagnets, in particular the 1,3,5-Triphenyl-6-oxoverdazyl (TOV) compound [16] and the $\square$ phase of the dithiadiazolyl radical $N C-\mathrm{C}_{6} \mathrm{~F}_{4}-\mathrm{CNSSN} \cdot[17,18]$, whose transition temperatures are $4.8 \mathrm{~K}$ and $36 \mathrm{~K}$ respectively. The magnitude of the spincanting is related to the magnetic anisotropy which, as we have already seen (section 1.1 ), is very small for organic free radicals. As a consequence, organic free radicals tend to exhibit very low canting angles and, consequently, very low values of the magnetization, e.g., $1.5 \square 10^{-3} \square_{\mathrm{B}} / \mathrm{mol}$ at $0 \mathrm{~K}$ are observed. (cf $1.0 \square_{\mathrm{B}} / \mathrm{mol}$ for organic ferromagnets built upon an equivalent $S=1 / 2$ spin system).

\subsubsection{Application to Purely Organic Systems}

The most obvious structure presenting spontaneous magnetization is the ferromagnetic one. However, only a few compounds have been reported as ferromagnetic. The first discovered examples were the already mentioned $p$-NPNN radical [1, 19 and references therein], the TDAE- $\mathrm{C}_{60}$ charge-transfer salt [20] and the diazaadamantane dinitroxyl biradical [21]. Recently, the discovery of two S-based ferromagnets have been reported: the dithiadiazolyl derivative $p-\mathrm{O}_{2} \mathrm{NC}_{6} \mathrm{~F}_{4} \mathrm{CNSSN}$ - [22] and the organic cation radical salt [BBDTA] $\left[\mathrm{GaCl}_{4}\right] . \mathrm{CH}_{3} \mathrm{CN}$ [23] with ferromagnetic transition temperatures of $1.3 \mathrm{~K}$ and $6.7 \mathrm{~K}$ respectively. The former radical belongs to the family of compounds studied in this chapter. The reason for the low number of ferromagnetic free-radical crystals is that antiferromagnetic interactions are usually more favourable. Whilst the magnetic interaction between radicals may be strongly ferromagnetic in two dimensions, a net antiferromagnetic coupling in the third dimension will lead to an antiferromagnetic ground state, i.e. very strict conditions must be met to obtain ferromagnetic interactions. 


\subsection{Dithiadiazolyl Radicals}

Almost all the attempts in the design of purely organic systems showing spontaneous magnetization have involved nitroxide $\mathrm{NO} \bullet$ radical derivatives as building blocks (see ref. [24] and contributions therein). In these radicals the spin delocalization over the $\mathrm{NO} \cdot$ groups provides high stability. Whilst the spin-bearing oxygen atoms are available at the molecular periphery, the electrostatically repulsive nature of the $\mathrm{O}^{\square} \square \mathrm{O}^{\square}$ contacts simultaneously disfavours close approach of regions of spin density. A systematic search of the Crystallographic database reveal no O $\square$ O contacts less than the sum of the van der Waals radii [6]. To date the highest ferromagnetic transition temperature of a nitroxide radical derivative is $1.48 \mathrm{~K}$ in the diazaadamantane dinitroxyl biradical [21].

One approach to raise the magnetic ordering temperature utilises the more diffuse nature of the $3 p$ sulphur orbitals compared to the $2 p$ ones of the oxygen atoms. Sulphurbased radicals should in principle favour stronger molecular interactions than oxygenbased ones $[25,26,27]$, since the more radially diffuse $3 p$ orbitals should facilitate interactions at greater distances ( $c f$ van der Waals radii of $\mathrm{O}$ and $\mathrm{S}$ at 1.50 and $1.74 \AA$ ). In addition $S / N$ based radicals favour electrostatically attractive $S^{\square} \square N^{\square}$ contacts and exhibit a multitude of $\mathrm{S} \square \mathrm{N}$ contacts less than the sum of the van der Waals radii.

The heterocyclic dithiadiazolyl radicals $\mathrm{RCN}_{2} \mathrm{~S}_{2}{ }^{\cdot}$ have provided promising results in the framework of this sulphur-based approach for obtaining purely organic ferromagnets, as it will be shown later. Of the two known isomers, the 1,3,2,4dithiadiazolyl radical, undergoes rearrangement to the thermodynamically more stable 1,2,3,5-dithiadiazolyl radical [28]. The latter have been the object of extensive studies $[29,30]$, particularly in relation to their potential application in the design of organic conductors via regular stacks of the heterocyclic rings. Rawson et al. [31] have reviewed the chemistry of the dithiadiazolyl radicals. This Chapter reviews the magnetic properties of these radicals.

The SOMO orbital in the 1,2,3,5-dithiadiazolyl radical is an antibonding $\square^{*}$ orbital localized on the heterocyclic ring with a node on the carbon atom due to the $a_{2}$ symmetry of the orbital (see Fig. 1) [32]. A very important consequence of the node at the carbon atom is that the electronic properties of this radical, e.g., hyperfine coupling constants, solution reduction and oxidation potentials, and gas phase ionization potentials show very little dependence on the $R$ substituent at the carbon atom. Therefore, these $R$ substituents can be varied in order to tune the crystal packing of the radicals for material applications without significantly changing the electronic properties of the dithiadiazolyl ring. Ab initio calculations have indicated a significant

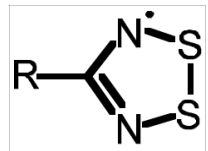

a)

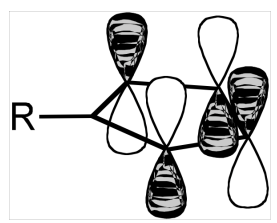

b)

Fig. 1. a) Scheme of the 1,2,3,5-dithiadiazolyl radical. b) SOMO orbital for the 1,2,3,5dithiadiazolyl radical 

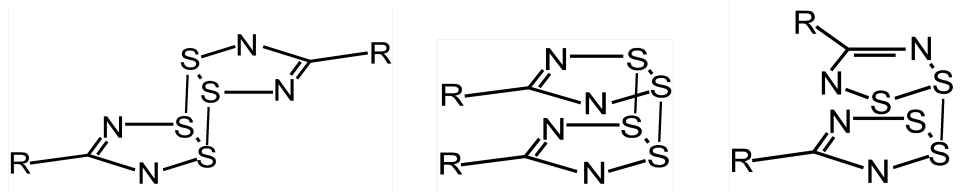

Fig. 2. Dimerization geometries of the dithiadiazolyl radicals.

polarity of the S-N bond, in the sense $\mathrm{S}^{\square^{+}} \square \mathrm{N}^{\square}$. Consequently, there will be a strong tendency for these radicals to pack in such a way as to maximise the intermolecular $\mathrm{S}^{\square+} \square \mathrm{N}^{\square}$ interactions. Since the $\mathrm{S}$ and $\mathrm{N}$ atoms bear the majority of the spin density (see Fig. $1 \mathrm{~b}$ and Section 2) then these close contacts should favour strong magnetic interactions. In addition the inclusion of sulphur generates additional spin-orbit coupling leading to a greater anisotropy than nitroxide radicals [33].

The main difficulty of utilising these radicals in the design of molecular magnetic materials is the lack of sufficient steric protection to inhibit dimerisation. Solution EPR studies on dithiadiazolyl radical derivatives in solution have determined a favourable dimerization enthalpy of $\sim 35 \mathrm{~kJ} / \mathrm{mol}$ [31]. Such a high dimerisation energy is due to the strong $\square-\square$ bonding interaction between the SOMO orbitals with close $\mathrm{S} \square \mathrm{S}$ and/or $\mathrm{N} \square \mathrm{S}$ and $\mathrm{N} \square \mathrm{N}$ contacts. The significant overlap between the SOMO orbitals from each radical in the dimer produces a spin-paired singlet ground state and therefore diamagnetic behaviour. Three typical dimerisation geometries of the dithiadiazolyl radicals are shown in Fig. 2.

Perfluoraryl groups have been proposed as the $\mathrm{R}$ substituents in order to avoid the dimerisation [31, 34] since i) the intramolecular N...F repulsions lead to a large twist angle between perfluorophenyl and dithiadiazolyl ring planes, making the phenyl group more sterically demanding; ii) the electrostatic repulsion between the perfluorophenyl rings may inhibit dimerisation; iii) the inclusion of strongly structure-directing groups. These may lead to structures in which the structure-directing group becomes the dominant intermolecular interaction and crystal growth utilising these functional groups may occur at the expense of the dimerisation process. This appears particularly successful when either the heterocyclic $\mathrm{N}$ or $\mathrm{S}$ atoms are themselves part of the structure-directing interaction, either through formation of favourable $S^{\square^{+}} \square X^{\square}$ or $N^{\square} \square X^{\square^{+}}$interactions.

To date, five perfluoroaryl-dithiadiazolyl radical crystals in which the dimerization tendency has been inhibited have been synthesized (see Fig. 3) [17, 22, 35, 36, 37, 38]. These five structures exhibit very different magnetic behaviour. One of the most interesting is the $p-\mathrm{NCC}_{6} \mathrm{~F}_{4} \mathrm{CNSSN} \bullet(\mathbf{1})$ which is polymorphic and crystallises in two different phases, $\square$ and $\square$. The $\square$ phase, $1 \square$, exhibits the highest transition temperature to a magnetic ordered phase which displays a spontaneous magnetization [18]. In this compound the magnetic interactions are antiferromagnetic but the magnetic space group allows the canting of the spins, which results in weak ferromagnetism (see Section 1.2.5). Both the $\square$ phase of the same dithiadiazolyl radical [35] and $p$ - $\mathrm{BrC}_{6} \mathrm{~F}_{4} \mathrm{CNSSN}$ (2) present paramagnetic behaviour with a negative value of the Weiss constant [39]. These negative Weiss constants indicate the existence of antiferromagnetic interactions 
between the radicals but an absence of long range order. Conversely the $p-\mathrm{O}_{2} \mathrm{NC}_{6} \mathrm{~F}_{4} \mathrm{CNSSN} \cdot$ radical (3) shows ferromagnetic interactions and a ferromagnetic transition at $1.32 \mathrm{~K}[22,40]$. The fourth compound, the $p-\mathrm{NCC}_{6} \mathrm{~F}_{4} \mathrm{C}_{6} \mathrm{~F}_{4} \mathrm{CNSSN} \cdot(4)$ exhibits a structure-directing $\mathrm{CN} \cdots \mathrm{S}$ interaction as observed in $1 \square$ and $1 \square$ but exhibits near perfect Curie paramagnet $(|\square|<1 \mathrm{~K})$ consistent with large distances between heterocyclic rings beyond $6 \AA$ [38]. The magnetic behaviour of the first four radicals will be explained in more detail later in this chapter. It should be emphasised that these dithiadiazolyl radicals are not the only dithiadiazolyl radicals to exhibit solid state paramagnetism (see also refs. $[41,42,43]$ ) but, as we shall see, their strong electronic and structural similarities provide an excellent group of molecules from which to draw

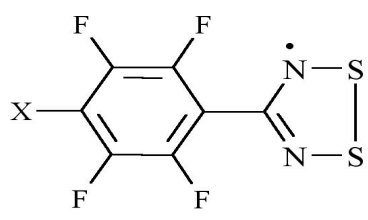

$1 \mathrm{X}=\mathrm{CN}$

$2 \mathrm{X}=\mathrm{Br}$

$3 \mathrm{X}=\mathrm{O}_{2} \mathrm{~N}$

$4 \mathrm{X}=\mathrm{NCC}_{6} \mathrm{~F}_{4}$

Fig. 3 Paramagnetic dithiodiazyl derivatives meaningful conclusions.

As seen in this general introduction to organic magnets, the unpaired electron distribution in the free radicals and the crystal packing play a crucial role in the magnetic behaviour of the free-radical solids. In fact, the important parameter is the spin density distribution, which not only reflects the unpaired electron distribution but also the polarization effects of these unpaired electrons on the doubly occupied molecular orbitals. A favourable packing to achieve long-range magnetic order must allow the proximity of the spin density of different free radicals and the propagation of the magnetic interactions through the solid. In addition, the nature of the magnetic interaction depends strongly on the relative geometry between the interacting magnetic orbitals which is also determined by the crystal packing. In other words, the different magnetic behaviour of the monomeric dithiadiazolyl radicals, even in two polymorphic crystals of the same radical, is due to the differences in their crystal packing. In the sections which follow we utilise radicals 1 $-\mathbf{3}$ to exemplify the sensitivity of the magnetic behaviour to variations in structure whilst retaining a constant spin density distribution.

\section{Spin Densities in the Dithiadiazolyl Ring}

The magnetic behaviour of several dithiadiazolyl radicals, including both polymorphs of 1 and the bromo derivative 2, have been studied in detail by Antorrena together with other thiazyl radicals [44]. The work also investigated the spin density in the perfluoroaryl derivatives of the dithiadiazolyl radical.

The spin density of dithiadiazolyl radicals has been undertaken from a theoretical perspective with semi-empirical and ab initio calculations and from an experimental point of view with EPR and Electron Spin Echo Envelope Modulation (ESEEM) spectroscopic techniques and with polarized neutron diffraction experiments.

The spectroscopic methods have several pecularities with respect to the neutron polarized diffraction experiments: i) they only provide estimates of how much of the spin density is associated with each spectroscopically active nucleus but does not provide information on the spatial density distribution about each nucleus; ii) EPR can only act as a reporter of the spin density distribution at those nuclei with non-zero 
nuclear spin e.g. ${ }^{14} \mathrm{~N}$ and ${ }^{19} \mathrm{~F}$; iii) the signal assignment is not always simple; iv) in EPR and ESEEM the sign of the spin density is not trivially determined; v) in EPR and ESSEM the experiments are typically performed in solution and reflect the spin density distribution in magnetically isolated ions not on the actual crystal structure (in the solid state dipolar line broadening often gives rise to line broadening which inhibits observation of the hyperfine interactions which reflect the spin density distribution); vi) antiferromagnetic compounds can be studied using EPR spectroscopy.

Different approaches to the experimental determination of the spin density in dithiadiazolyl derivatives using continuous-wave EPR (CW-EPR) and ESEEM spectroscopies have been carried out $[31,45)$. Only the local spin population at the heterocyclic nitrogen atom and the aromatic fluorine atoms could be investigated with these techniques. In addition to these experimental results, there are also ab-initio calculations of the Mulliken spin populations [18,44]. All these studies suggest that the spin density is located basically on the dithiadiazolyl ring and it is almost independent of the $R$ substituent. Hence, the spin density distribution in the dithiadiazolyl ring for the different derivatives should be very similar to each other. Therefore, the spin density distribution obtained experimentally from one compound can be extrapolated to the other ones.

Unlike resonance techniques, polarized neutron diffraction (PND) provides a direct probe of the actual spatial distribution of the spin density in the solid. This powerful method has already been applied to other purely organic compounds [46, 47, 48], where it has contributed significantly to the understanding of their magnetic behaviour.

\section{Spin Density Studies on the p-nitro-tetrafluorophenyl- dithiadiazolyl radical (3)}

The ideal situation for a PND experiment is to have all the spins parallel. Hence $1 \square$ is not suitable for this kind of experiments because of its antiferromagnetic phase transition at $36 \mathrm{~K}$. The magnetic field necessary to polarize significantly the spins at low temperature is much higher than the accessible laboratory magnetic field (about 10 Tesla). Among the other dithiadiazolyl radicals, 3 was chosen for the PND experiment [49] for several reasons: i) single crystals up to $1-2 \mathrm{~mm}^{3}$ were available; ii) the other radicals present antiferromagnetic interactions, which hinders the induced spin polarization; iii) this radical is intrinsically interesting due to its ferromagnetic transition at $1.32 \mathrm{~K}$. In the

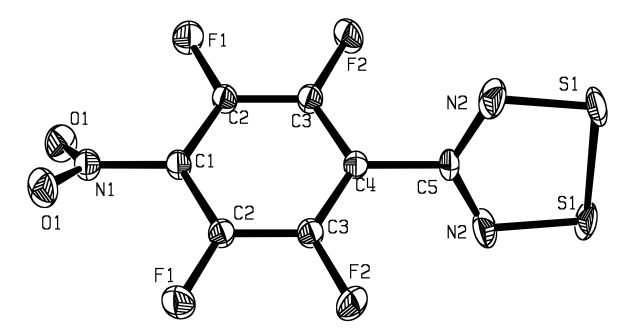

Fig. 4. View of the $p-O_{2} \mathrm{NC}_{6} \mathrm{~F}_{4} \mathrm{CNSSN} \cdot$ molecule determined by single-crystal neutron diffraction experiments at $20 \mathrm{~K}$. The thermal ellipsoids are at the $99 \%$ probability level. 
following sections the crystallographic and magnetic behaviour of this compound will be described with some detail before discussing the PND results.

\subsection{Crystal Structure and Magnetic behaviour of the p- $\mathrm{O}_{2} \mathrm{NC}_{6} \mathrm{~F}_{4} \mathrm{CNSSN} \bullet$ Radical}

The crystal structure of $\mathbf{3}$ has been determined by X-ray and neutron diffraction at, respectively, 180 and $20 \mathrm{~K}$ (Fig. 4) [22, 49]. A 180K, this radical crystallizes in a noncentro symmetric tetragonal structure, space group $P 4_{1} 2_{1} 2$, with four molecules per unit cell and with the following cell parameters: $a=8.138 \AA, c=15.049 \AA$ (Fig. 5a). Molecules are arranged in sheets perpendicular to the $c$ axis rotated 90 degrees with respect to each other. In these sheets, the molecules form chains through electrostatic $\mathrm{S}^{\square} \square \mathrm{O}^{\square}$ interactions and these chains are linked via intermolecular O...F contacts in the $a b$ plane (Fig. 5b). These sheets are related by a 4 -fold screw axis parallel to the $c$ axis, with electrostatically favourable inter-layer $S^{\square+} \square N^{\square}$ contacts. There is no crystallographic transition is observed in the whole temperature range from room temperature to $1.5 \mathrm{~K}$. The cell parameters at $20 \mathrm{~K}$ reflect a slight contraction of the cell upon cooling ( $a=8.1125(6) \AA, c=14.7646(9) \AA)$.

With the exception of BBDTA- $\mathrm{GaCl}_{4}-\mathrm{CH}_{3} \mathrm{CN}$ [23], 3 is the only S-based free radical derivative that has been described as a pure ferromagnet [22]. The high temperature magnetic susceptibility shows a Curie-Weiss behaviour with $\square_{\mathrm{cw}}=1.6 \pm 0.1 \mathrm{~K}$. The positive value of the Curie-Weiss constant indicates the presence of ferromagnetic interactions between the $1 / 2$ spin centres. Indeed, the low temperature $a c$ magnetic susceptibility on a single crystal exhibits a ferromagnetic transition at $1.32 \pm 0.02 \mathrm{~K}$ (Fig. 6). In the magnetic measurements small anisotropy is observed as expected for a compound where the only possible source of magnetic anisotropy is the magnetic dipolar interactions between the radicals.

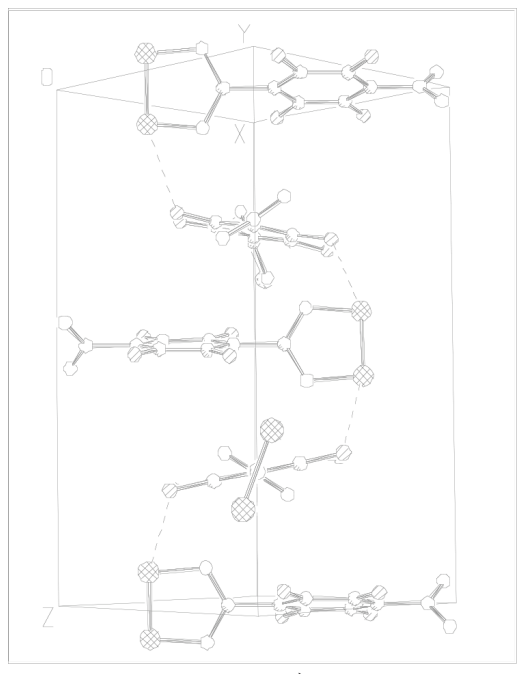

a)

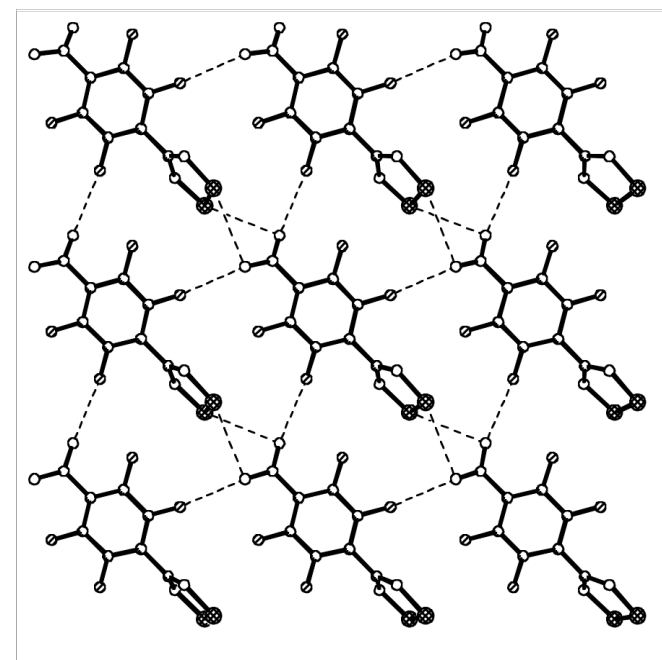

b)

Fig. 5. a) The four molecules in the unit cell of 3. b) A sheet of 3 in the $a b$ plane. 


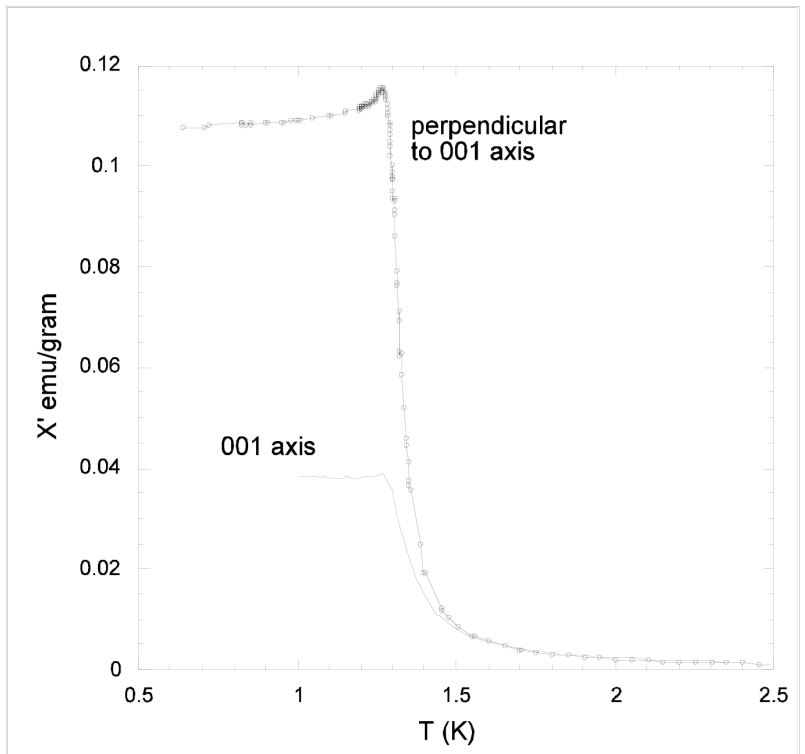

Fig. 6. Single crystal magnetic $a c$ susceptibilities $(\square=1.11 \mathrm{~Hz}) v s$ temperature in $\mathbf{3}$ for the $(001)$ and perpendicular to the (001) orientations of the crystal with respect to the $a c$ field.

\subsection{Polarized Neutron Diffraction Experiments on the p- $\mathrm{O}_{2} \mathrm{NC}_{6} \mathrm{~F}_{4} \mathrm{CNSSN} \cdot$ Radical}

The same crystal of $\mathbf{3}$ that was used in the structure determination at $20 \mathrm{~K}$ was used for the polarized neutron diffraction experiments. The magnetic saturation of the sample was achieved by the application of magnetic field of 9 Tesla at $1.5 \mathrm{~K}$ [49].

In a non-centrosymmetric crystal structure the nuclear and magnetic structure factors are complex numbers and the magnetic structure factors must be calculated from analytical modelling of the spin density distribution in order to fit the experimental flipping ratios. The wavefuntion [50, 51] and the multipolar expansion [46] are the two different approaches commonly used for modelling the spin density distribution. Whereas the wavefunction approach provides a straightforward interpretation of the physical origin of the spin density distribution, the multipole approach confers more flexibility to the model, enlighting details which cannot be deduced from the wavefunction approach in a simple manner. For complementarity, results from both approaches will be discussed in this chapter in order to provide an accurate picture of the spin density distribution in the radical.

Several models considering different number of parameters and different constraints were used to calculate the magnetic form factors within each approach. In the case of the wavefunction approach the model providing the best results included the $p_{z}$ orbitals of the carbon, nitrogen and sulphur atoms of the heterocyclic ring together with the $p_{x}$ and $p_{y}$ orbitals of the nitrogen and sulphur atoms and a coefficient for the $2 s$ nitrogen orbital to account for the $s p^{2}$ hybridation of the nitrogen atoms. The $2 p_{z}$ orbital was the only orbital considered for the carbon atom because preliminary Density Functional Theory (DFT) calculations [44] indicated that its spin population is one order of magnitude lower than the sulphur and nitrogen ones. More simplified models, like ignoring the $2 s$ orbital in the nitrogen atoms, give poorer agreement factors. Instead, the multipolar expansion approach seems to be less sensitive and a simple model assuming that the plane of the heterocyclic ring is a symmetry plane, hence considering only even 
multipoles in $z$, provided the best results with the minimum number of parameters. Calculations using more complex models including odd multipoles in $z$ and the carbon atoms in the aromatic ring provided essentially the same results.

The main results from both approaches are compared in Table 1, where the total spin density population on the atoms of the dithiadiazolyl ring is normalised to $1 \square_{\mathrm{B}}$ per radical, the sum of the populations before the normalisation is represented by $m\left(\square_{\mathrm{B}}\right)$ which corresponds to the magnetisation of the radical, the number of parameters of the model is represented by $n_{v}$ and $\square^{2}$ is the agreement factor of the fit.

Figure 7 and 8 give several projections of the spin density distribution in relation to the atoms of the dithiadiazolyl ring as calculated following the wavefunction and the multipolar expansion approach, respectively. The results from both approaches indicate that the spin density is almost entirely localized in $p_{z}$ orbitals of the nitrogen and sulphur atoms of the dithidiazolyl radical. This is consistent with the SOMO nature: an antibonding $\square$ orbital extended on the sulphur and nitrogen atoms. Beside this spin density coming from the SOMO orbital, there exists some $p_{z}$ spin density on the carbon atom induced by spin polarization. As for the spin density populations, they are in agreement with previous values from the resonance experiments and $a b$-initio calculations.

The negative spin density at the carbon atom is due to a polarisation effect produced by the SOMO orbital on some of the doubly occupied orbitals. In the dithiadiazolyl ring there are seven electrons in $\square$ orbitals, six in three doubly occupied molecular orbitals and one in the SOMO orbital. Among the three doubly occupied orbitals there are two with $b$ symmetry, and therefore without a node at the carbon atom. In these orbitals the $\square$ orbital (spin down) will be more localized in the carbon atom than the $\square$ orbital (spin up) in order to avoid the electrostatic repulsion with the electron in the SOMO orbital. In the $\square$ orbitals the electrostatic repulsion is in certain way compensated by the exchange interaction between electrons with parallel spins. This spatial difference between the $\square$ and $\square$ orbitals is the origin of the negative spin density at the carbon atom.

In the wavefunction approach a canting can be clearly observed in the projection of the sulphur and nitrogen spin density distribution on the $y z$ plane (Fig. 7). Besides the possibility that the wavefunction approach is not completely well adapted to describe the experimental spin density due to its constraints, the physical origin of this canting can be attributed to the proximity of the fluorine atom in the ortho position of the perfluoraryl ring. Due to the torsion between the two rings, this fluorine atom is not in the plane of the heterocyclic ring but closer to one of the lobes of the nitrogen $3 p_{z}$ orbital The polarisation of the electron density away from nitrogen by the electronegative

\section{Table 1}

Comparative results of the spin density population on the atoms of the dithiadiazolyl ring, magnetisation of the radical, $m\left(\square_{\mathrm{B}}\right)$, number of parameters used, $n_{v}$, and agreement factor for the two approaches followed to analyse the PND results.

\begin{tabular}{|c|c|c|c|c|c|c|}
\hline Approach & \multicolumn{3}{|c|}{ Population $\left(\square_{\mathrm{B}}\right)$} & $m\left(\square_{\mathrm{B}}\right)$ & $n_{v}$ & $\square^{2}$ \\
\hline & $\mathrm{S}$ & $\mathrm{N}$ & $\mathrm{C}$ & & & \\
\hline Wavefunction & $0.301(1)$ & $0.222(1)$ & $-0.046(1)$ & $0.894(2)$ & 9 & 2.33 \\
\hline $\begin{array}{l}\text { Multipolar } \\
\text { expansion }\end{array}$ & $0.284(1)$ & $0.242(1)$ & $-0.053(2)$ & $0.923(3)$ & 21 & 1.29 \\
\hline
\end{tabular}




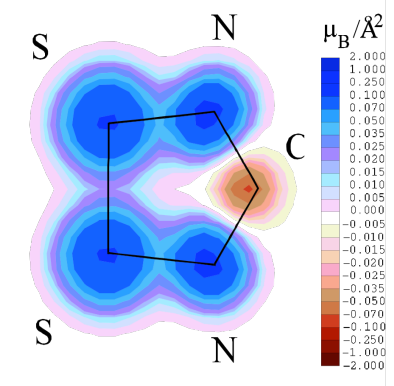

a)

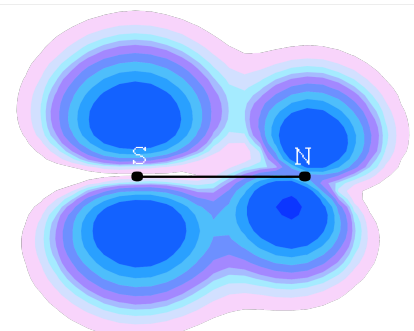

b)

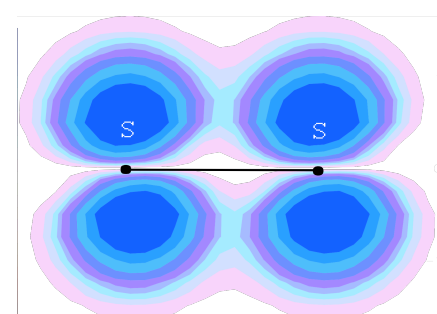

c)

Fig. 7. Projection of the calculated spin density distribution following the wavefunction approach: a) onto the plane containing the dithiadiazolyl ring; b) in a plane perpendicular to the dithiadiazolyl ring containing $\mathrm{S}$ and $\mathrm{N} ; \mathrm{c}$ ) in a plane perpendicular to the dithiadiazolyl ring containing $\mathrm{S}$ and $\mathrm{S}$.

fluorine may well be the origin of the canting and is supported by EPR studies and DFT which reflect some unpaired spin density at the ortho-fluorine atoms.

The multipolar expansion approach provides two important features of the spin density distribution that cannot be present in the wavefunction approach because of its constraints. The first is the displacement of the sulphur spin density away from the sulphur and nitrogen neighbouring atoms. This effect is clearly observed in Fig. 8, where the projection of the sulphur spin density is not centred on the sulphur atom but is displaced away from the centre of the ring. This displacement of the spin density is consistent with the anti-bonding nature of the SOMO orbital: while in the bonding molecular orbitals there is a concentration of electronic density in the bond, in the antibonding one the electronic density is away from the bonds. The second feature in these models is the negative spin density between the sulphur atoms and between the sulphur and nitrogen atoms. This negative spin density could be due to polarization effects produced by the SOMO orbital on the other double occupied molecular orbitals, although it could also be a spurious effect of the models trying to fit correctly the spin density displacement in the sulphur atom. A similar spurious effect has been noted in the PND spin density distribution in a nitronyl nitroxide radical [52].

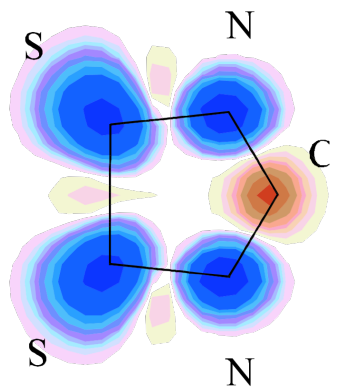

a)

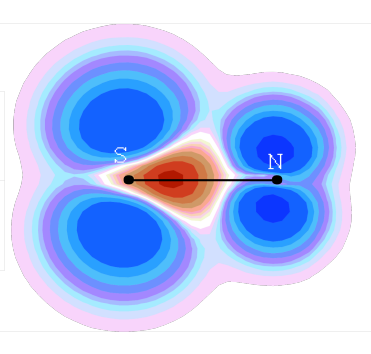

b)

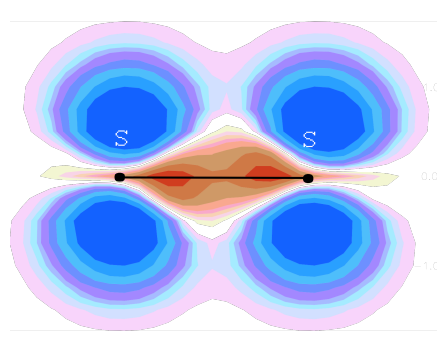

c)

Fig. 8. Projection of the calculated spin density distribution following the multipolar expansion approach: a) onto the plane containing the dithiadiazolyl ring, b) onto a plane perpendicular containing both $\mathrm{S}$ and $\mathrm{N}$ atoms and $\mathrm{c}$ ) containing both $\mathrm{S}$ atoms. 
Table 2.

DFT Mulliken spin populations in $\mathbf{3}$

\begin{tabular}{lrrrrr}
\hline Atom & \multicolumn{2}{c}{ DMOL3 } & \multicolumn{3}{c}{ Gaussian98 } \\
& Periodic & Radical & \multicolumn{1}{c}{ PW91 } & B3LYP & \multicolumn{1}{l}{ UHF } \\
\hline S1 & 0.320 & 0.311 & 0.2910 & 0.2787 & 0.1390 \\
N2 & 0.213 & 0.224 & 0.2455 & 0.2868 & 0.6623 \\
C5 & -0.063 & -0.065 & -0.0667 & -0.1252 & -0.6238 \\
C4 & 0.005 & 0.005 & 0.0003 & 0.0078 & -0.0446 \\
C3 & -0.003 & -0.004 & -0.0030 & -0.0069 & 0.0840 \\
C2 & 0.002 & 0.001 & 0.0014 & 0.0036 & -0.0993 \\
C1 & -0.003 & -0.003 & -0.0033 & -0.0067 & 0.0974 \\
F2 & 0.000 & 0.000 & 0.0003 & 0.0000 & 0.0014 \\
F1 & 0.000 & 0.000 & 0.0001 & 0.0002 & -0.0007 \\
N1 & 0.001 & 0.000 & 0.0002 & 0.0005 & -0.0130 \\
O1 & 0.000 & 0.000 & -0.0005 & -0.0007 & 0.0053 \\
\hline
\end{tabular}

The fact that calculations including the carbon atoms in the aromatic ring provided essentially the same results indicate that there is no significant spin population away from the dithiadiazolyl ring. The population in these carbon atoms is, indeed, one order of magnitude less than the population in the carbon atom of the dithiadiazolyl ring.

In conclusion, the PND experiments indicate that the spin density is almost entirely located in the sulphur and nitrogen atoms of the dithiadiazolyl ring in a $p_{z}$-orbital-type distribution with the $z$ axis perpendicular to the ring. This spin density arises from the anti-bonding nature of the SOMO orbital and is displaced from the centre of the atoms outside the bonds This displacement is most pronounced at the sulphur atoms, which will favour through space magnetic interactions with other radicals.

\subsection{Theoretical Spin Densities}

PND results have been complemented with theoretical computation of the spin density distribution using two different ab initio packages, the DMOL3 package and the Gaussian98 package [53]. The geometry obtained from the low-temperature nonpolarized neutron experiment was used in all the calculations [54].

The Mulliken spin populations calculated for all the atoms of $\mathbf{3}$ using both packages are listed in Table 2. (Note that the molecule sits on a two-fold rotation axis. The atom numbering scheme is presented in Fig. 4). They are in good agreement with the PND results, confirming that all the spin density is located on the dithiadiazolyl ring. Moreover, the spin density distribution from the ab-initio calculation confirms the $p_{z}$ nature of the spin density and the spin density displacement for the sulphur atoms. In addition there is also a negative spin density distribution in the plane of the dithiadiazolyl ring, as can be observed in Fig. 9. The value of this negative spin density is however much smaller than the negative spin density observed in the PND experiment. 


\section{Magnetic Interactions in Dithiadiazolyl Radicals}

The magnetic interactions of the closely-related group of monomeric dithiadiazolyl radicals, $p-\mathrm{XC}_{6} \mathrm{~F}_{4} \mathrm{CNSSN} \cdot$, where $\mathrm{X}=\mathrm{CN}(\mathbf{1}), \mathrm{Br}(\mathbf{2}), \mathrm{O}_{2} \mathrm{~N}(\mathbf{3})$ are discussed in this section. Since all the molecules differ only in the group linked to the tetrafluorophenyl ring, where there is no measurable spin density, they are all magnetically similar. When these molecules are assembled in the solid state, they exhibit very different intermolecular contacts. As a consequence their magnetic properties turned to be very different from one another. Theoretical calculations show that the dominant exchange interactions between these thiazyl radical groups are through direct exchange processes and emphasise the extreme influence that the molecular packing has on the bulk magnetic properties of the compound [55].

In recent years DFT has proved to be an extremely powerful approach to the determination of the exchange interaction in both metal clusters [56] and through space interactions in organic radicals. Indeed systematic studies by Novoa and Yamaguchi (see chapter in this book and references therein) have shown that the bulk magnetic behaviour of organic solids can be determined through an evaluation of the local nearest-neighbour exchange interactions between radicals [6]. In particular, Novoa's studies on aryl-substituted nitronyl nitroxide radicals clearly showed that whilst the phenyl substituent itself bore negligible spin density, it contributed significantly to the magnetic exchange pathway.

The general approach to determine the magnetic exchange interaction between two nearest neighbour radicals is to compute the energies of the triplet $\left(\mathrm{E}_{\mathrm{T}}\right)$ and broken symmetry singlet $\left(\mathrm{E}_{\mathrm{BS}}\right)$ states. The broken symmetry singlet state is a DFT state composed of pure magnetic states in which all the $\square$-magnetic orbitals are located on one magnetic center and all the $\square$-magnetic orbitals are located on the other magnetic center [57, 58, 59].

Within the context of the Hamiltonian $\mathrm{H}=-2 \boldsymbol{J} \mathbf{S}_{1} \cdot \mathbf{S}_{2}$, where $\boldsymbol{J}$ is the exchange interaction between spins $S_{1}$ and $S_{2}$, the energy between the singlet and triplet is denoted by $2 \boldsymbol{J}$. Yamaguchi proposed [60] that the exchange interaction could be estimated from the energies of the broken symmetry singlet and triplet states and their expectation values $<\mathrm{S}^{2}>$, according to the expression:

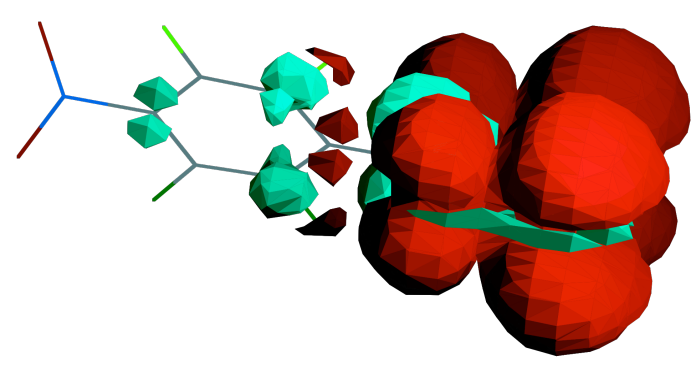

Fig. 9. Two spin density isosurfaces, from the molecular DMOL3 Perdew-Wang 91 calculation, for a value of $2 \square 10^{-4} \square_{\mathrm{B}} \AA^{3}$ in modulus. Red and blue areas refer to regions of positive and negative spin density respectively. 
$J=\square \frac{E_{H S} \square E_{B S}}{\left\langle S^{2}\right\rangle_{H S} \square\left\langle S^{2}\right\rangle_{B S}}$

Density functional calculations were carried out using the hybrid exchange correlation functional B3LYP which has proved to correct the tendency of the local and GGA exchange correlation functionals to overestimate the stability of the singlet state with respect to the triplet [61]. Two different basis sets were employed: the polarized split-valence double-zeta $\left(6-31 \mathrm{G}^{* *}\right)$ and triple-zeta $\left(6-311 \mathrm{G}^{* *}\right)$ Pople basis sets [62] in order to test the convergence of the calculation with the size of the basis set. Values of $|\boldsymbol{J}|$ were considered significant when $|\boldsymbol{J}|>1 \mathrm{~cm}^{-1}$.

In the following of the section the geometry of all the nearest neighbour interactions within the structures of the three above mentioned $p$-tetrafluorophenyl derivatives will be discussed together with their respective magnetic properties. Since the magnetic interaction depends on the distance $r$ between the magnetic centres as $\exp (-r)$ [63], possible magnetic pathways are selected within two cut-off distances. As almost all the spin density is located on the dithiadiazolyl ring, selected radical pairs are with an intermolecular distance shorter than $7 \AA$ between atoms in both dithiadiazolyl rings. Moreover, since it has been demonstrated that magnetic interactions can be transmitted through atoms with very low spin populations [64], radical pairs with an atom of the dithiadiazolyl ring in one radical and an atom in a neighbour radical at a distance shorter than $4 \AA$ are also considered. This methodology for determining all the possible magnetic pathways is similar to the Deumal-Novoa methodology [65].

\subsection{Exchange Interactions in $\mathrm{p}-\mathrm{O}_{2} \mathrm{NC}_{6} \mathrm{~F}_{4} \mathrm{CNSSN} \bullet$}

As explained in section 2.1, radical 3 is one of the rare examples of a bulk organic ferromagnet with a Curie temperature of $1.32 \mathrm{~K}$ at ambient pressure. Its behaviour under applied pressure is also unusual for most of the organic ferromagnets investigated exhibit a decrease in Tc as pressure increases whereas the opposite occurs in antiferromagnets and weak ferromagnets [66]. In the case of $\mathbf{3}$ the ordering temperature raises up to 1.8K under $11.6 \mathrm{kbar}$ [67]. The structure of $\mathbf{3}$ has been described in section 2.1. Four magnetic interaction pathways can be identified within the rules explained above. They are represented in the scheme of Fig. 10:

- $J_{1}$ : This pathway connects a radical with four other radicals, two in the upper $a b$ plane and two in the lower $a b$ plane through heterocyclic $\mathrm{S} \cdots \mathrm{N}$ contacs of $3.658 \AA$, the shortest between atoms of neighbour dithiadiazolyl rings.

- $J_{2}$ : The $J_{2}$ pathway presents a similar structure of the interaction propagation to $J_{1}$, although in this case the shortest contact between atoms of neighbour dithiadiazolyl rings is very large, $6.989 \AA$. However, there are several intermolecular contacts between other atoms and the dithiadiazoyl ring shorter than $4 \AA$ that can potentially contribute to propagate magnetic interactions.

- $J_{3}$ : This pathway links the radical with its four nearest neighbours along the $a$ and $b$ axes, where the most relevant contact is between the sulphur of the dithiadiazolyl ring and a fluorine atom in meta position at $3.907 \AA$, just below the cut-off limit. 
- $J_{4}$ : Like $J_{3}$, this pathway is in the $a b$ plane and it propagates magnetic interactions through the chains formed by electrostatic interactions between the $\mathrm{NO}_{2}$ group and the sulphur atoms with $\mathrm{O} \cdots \mathrm{S}$ distances of $3.186 \AA$ and $3.323 \AA$.

The only exchange pathway connecting neighbouring dithiadiazolyl rings is $J_{l}$. Thus, $J_{l}$ should be much stronger than the other three magnetic interactions. This interaction propagates in a three-dimensional network, thus allowing a magnetic transition without the contribution of other magnetic interactions. Since this compound undergoes a ferromagnetic transition at $1.32 \mathrm{~K}$, the interaction must be ferromagnetic.

A theoretical analysis of the exchange interactions indicates that the strongest exchange coupling is via this $\mathrm{S}$... $\mathrm{N}$ contact and is weakly ferromagnetic $\left(\boldsymbol{J}_{1}=+1.14 \mathrm{~cm}^{-1}\right)$. All other interactions including those formed via the $\mathrm{NO}_{2} \ldots \mathrm{S}$ contact $\left(\boldsymbol{J}_{4}=-0.03 \mathrm{~cm}^{-1}\right)$ are more than an order of magnitude smaller (see Table 3 for more detailed results). A simple analysis of these interactions predict Weiss constants of $+1.80 \mathrm{~K}$ and $+1.64 \mathrm{~K}$ for the $6-31 \mathrm{G}^{* *}$ and $6-311 \mathrm{G}^{* *}$ basis sets respectively. Within the mean-field approximation, this leads to an upper limit for the magnetic ordering temperature of $1.80-1.64 \mathrm{~K}$, in excellent agreement with the observed value of $1.3 \mathrm{~K}$ [22].

It is interesting to notice that since the spin populations on the sulphur and nitrogen atoms in both dithiadiazolyl rings are positive, the $J_{1}$ interaction should be antiferromagnetic on the basis of the McConnell I mechanism. However, the McConnell I mechanism is a simplified model based on phenomenological observations and is not theoretically rigorous [5]. This model only considers the spin density populations, neglecting other factors such as the geometrical orientation of the magnetic orbitals. In order to understand the possible ferromagnetic nature of the $J_{l}$ pathway, we recall some points about the mechanisms of the magnetic interactions. As explained in Section 1.2, the magnetic interaction between electrons in two magnetic orbitals can be decomposed into two principal terms, one antiferromagnetic and the other ferromagnetic. In general, the antiferromagnetic term dominates, resulting in an antiferromagnetic interaction. This antiferromagnetic component depends linearly on the overlap between the magnetic orbitals. Consequently, if the magnetic orbitals are orthogonal the antiferromagnetic component vanishes and the resulting magnetic interaction is ferromagnetic. This "orthogonality method" is one of the methods used in molecular magnetism in order to obtain ferromagnetic interactions.

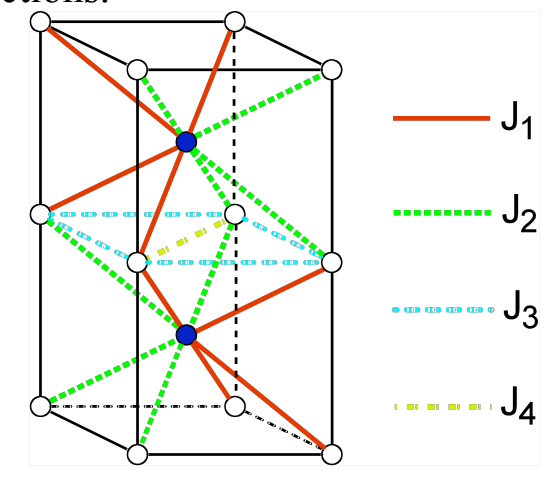

Fig. 10. Scheme of the possible magnetic pathways of $\mathbf{3}$ in the unit cell. The radical rings are represented by spheres. For clarity, the interactions in the $a b$ plane are only described in the plane $z=0.5$. 


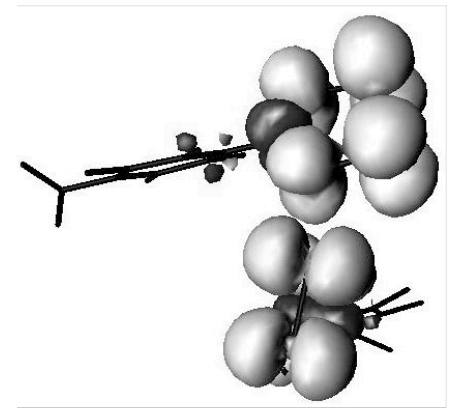

Fig. 11. Spin density isosurfaces for the $J_{1}$ radical dimer computed by DFT. Light grey isosurface is at $0.001 \square_{\mathrm{B}} \AA^{3}$ and the black isosurface is at $-0.001 \square_{\mathrm{B}} \AA^{3}$.

Table 3.

Broken Symmetry DFT magnetic coupling constants for 3.

\begin{tabular}{ccccc}
\hline Pathway & Contact & $d(\AA)$ & $\begin{array}{c}J\left(\mathrm{~cm}^{-1}\right) \\
\left(6-31 \mathrm{G}^{* *}\right)\end{array}$ & $\begin{array}{c}J\left(\mathrm{~cm}^{-1}\right) \\
\left(6-311 \mathrm{G}^{* *}\right)\end{array}$ \\
\hline$J_{1}$ & $\mathrm{~S} \cdots \mathrm{N}$ & 3.658 & +1.26 & +1.15 \\
$J_{2}$ & $\mathrm{~S} \cdots \mathrm{N}$ & 6.989 & +0.05 & +0.04 \\
$J_{3}$ & $\mathrm{C}_{1} \cdots \mathrm{N}_{2}$ & 3.503 & & -0.03 \\
$J_{4}$ & $\mathrm{~S} \cdots \mathrm{F}$ & 3.907 & -0.03 & -0.04 \\
\hline
\end{tabular}

Exchange interactions are based upon the crystal data at $160 \mathrm{~K}$.

Both radicals involved in the $J_{1}$ pathway are represented in Fig. 11. Although the SOMOs of both radicals are not strictly orthogonal by symmetry, their overlap should be very low because of their relative geometry. In the closest intermolecular contact $\mathrm{N} 2-\mathrm{S} 1$, one of the $2 p_{z}$ lobes of the nitrogen atom is pointing to the sulphur atom almost perpendicular to its $3 p_{z}$ orbital. This near orthogonality between the $p_{z}$ orbitals taking part in the magnetic interaction pathway favours the ferromagnetic part against the antiferromagnetic one in the magnetic interaction. Furthermore, the negative spin density in the plane of the ring would also reinforce the ferromagnetism due to the McConnell I mechanism. However, since this negative density is very low, according to the DFT calculations, its contribution to the ferromagnetic interaction will be of second order.

\subsection{Exchange Interactions and Magnetic behaviour of $\square-p-N C C_{6} F_{4} C N S S N \cdot$}

The $\square$-phase of $p$ - $\mathrm{NCC}_{6} \mathrm{~F}_{4} \mathrm{CNSSN}$ (1口) was the first reported dithiadiazolyl radical to retain its monomeric nature in the solid state [35]. Preliminary magnetic measurements on a Faraday Balance exhibit a broad maximum in $\square$ around $8 \mathrm{~K}$ indicative of short range antiferromagnetic interactions. Above $100 \mathrm{~K}$, the susceptibility follows a CurieWeiss law with $\square=-25 \mathrm{~K}$.

The structure of $1 \square$ is triclinic, space group $P-1$, with the two molecules per unit cell related by an inversion centre [35]. The low symmetry of the triclinic crystal system leads 
to a more varied range of close intermolecular contacts. The shortest of these are $\mathrm{CN}^{\square}$ $\ldots \mathrm{S}^{\square^{+}}$interactions which link radicals together into molecular chains $\left(d_{\mathrm{CN} \cdots \mathrm{S}}=3.068 \AA\right.$ and $3.105 \AA$ ). An additional web of S...S contacts links chains in an antiparallel fashion in the $a b$ plane $\left(\mathrm{d}_{1}=3.601, \mathrm{~d}_{2}=3.668 \AA\right)$. Along the $c$-axis a pair of close-heterocyclic $\mathrm{S} \cdots \mathrm{N}$ contacts $\left(\mathrm{d}_{\mathrm{S} . \ldots \mathrm{N}}=4.186\right.$ and $\left.4.269 \AA\right)$ form a centrosymmetric dimer. These loosely associated dimers are linked via $\mathrm{N} \cdots \mathrm{N}$ contacts $(5.064 \AA)$ about a crystallographic inversion centre. In Fig. 12 2 $\square 2 \square 2$ unit cells of $1 \square$ are viewed along the $a$ axis.

An analysis of all nearest neighbour exchange interactions in 10 reveals two significant interactions which alternate in a linear chain and comprise competing antiferromagnetic $\left(\boldsymbol{J}_{1}\right)$ and ferromagnetic $\left(\boldsymbol{J}_{2}\right)$ interactions of similar magnitude. Interestingly enough, a third interaction through the shorter of the two intermolecular $\mathrm{S} \cdots \mathrm{S}$ contacts in the $a b$ plane $\left(\boldsymbol{J}_{3}\right)$ propagates a negligibly small exchange coupling. These three shortest contacts, $\boldsymbol{J}_{1}, \boldsymbol{J}_{2}$ and $\boldsymbol{J}_{3}$, are pictured for one radical in Fig. 12. The data presented in Table 4 indicate that the determination of the exchange interactions has not fully converged at the $6-311 \mathrm{G}^{* *}$ level thus leaving some uncertainty in their respective magnitudes, although it is clear that $\boldsymbol{J}_{1}$ and $\boldsymbol{J}_{2}$ are the dominant interactions and propagate throughout the crystal lattice to generate a one-dimensional chain structure with alternating ferromagnetic and antiferromagnetic interactions. Nevertheless, the alternating signs of two interactions along the chain produces an expected upper limits

Table 4.

Broken Symmetry DFT magnetic coupling constants for $1 \square$.

\begin{tabular}{ccccc}
\hline Pathway & Contact & $d(\AA)$ & $\begin{array}{c}J\left(\mathrm{~cm}^{-1}\right) \\
\left(6-31 \mathrm{G}^{* *}\right)\end{array}$ & $\begin{array}{c}J\left(\mathrm{~cm}^{-1}\right) \\
\left(6-311 \mathrm{G}^{* *}\right)\end{array}$ \\
\hline$J_{1}$ & $\mathrm{~S} \cdots \mathrm{S}$ & 3.682 & -6.02 & -8.80 \\
$J_{2}$ & $\mathrm{~S} \cdots \mathrm{N}$ & 4.186 & +9.15 & +7.58 \\
$J_{3}$ & $\mathrm{~S} \cdots \mathrm{N}$ & 4.269 & & -1.00 \\
\hline
\end{tabular}

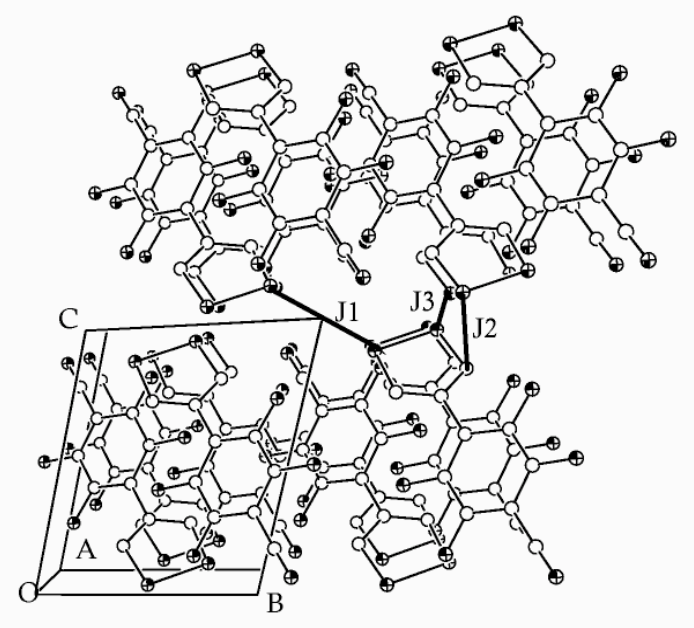

Fig. 12. $2 \square 2 \square 2$ unit cells of the $\square-N C-\mathrm{C}_{6} \mathrm{~F}_{4}-\mathrm{CNSSN} \cdot$ radical viewed along to the $a$ axis. The $J_{l}$, $J_{2}$ and $J_{3}$ pathways are represented for one radical. 


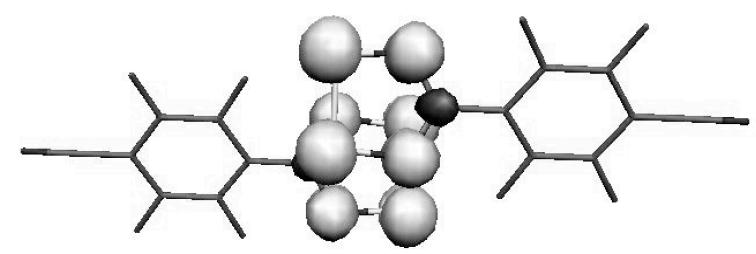

Fig. 13. Spin density isosurfaces for the $J_{1}$ radical dimer of the $\square-p-N C C_{6} \mathrm{~F}_{4} \mathrm{CNSSN}$ - radical. Light grey isosurface is at $0.01 \square_{\mathrm{B}} \AA^{3}$ and the black isosurface is at $-0.01 \square_{\mathrm{B}} \AA^{3}$. The plane of the view is parallel the dithiadiazolyl ring of the nearest radical.

for the short-range maximum in the susceptibility of a few Kelvin, somewhat less than the experimental value ( $c a .8 \mathrm{~K})$. This inconsistency merits further experimental studies.

The $\boldsymbol{J}_{2}$ interaction, which is ferromagnetic and stronger than the one that develops ferromagnetism in $\mathbf{3}$, deserves some discussion. . Due to the symmetry the SOMO orbitals in the $J_{l}$ pair are almost orthogonal, thus favouring a ferromagnetic interaction. Moreover, there is an overlap between the positive spin density of a sulphur atom and the negative spin density of the carbon atom in the dithiadiazolyl ring, which also favours a ferromagnetic interaction. The spin density isosurfaces are pictured in Fig. 13.

\subsection{Exchange Interactions and Magnetic behaviour of $\square-\mathrm{NC}-\mathrm{C}_{6} \mathrm{~F}_{4}-\mathrm{CNSSN} \bullet$}

The $\square$ phase of the $p-N C C_{6} \mathrm{~F}_{4} \mathrm{CNSSN} \bullet$, 1 $\square$., is one of the most extensively studied organic magnets. In this phase molecules of 1 crystallize in the non-centrosymmetric group $F d d 2$ with eight molecules per unit cell. As in the $\square$ phase, the crystal packing is governed by the electrostatic interactions between the sulphur atoms of the heterocyclic ring and the nitrogen atom of the cyano group producing a chain-like motif along the $c$ axis with short $\mathrm{CN}^{\square} \ldots \mathrm{S}^{\square^{+}}$contacts $(2.986 \AA)$. Contrary to the $\square$ phase, the chains are packed in a parallel way with favourable electrostatic interchain $\mathrm{S} \cdots \mathrm{N}$ interactions.

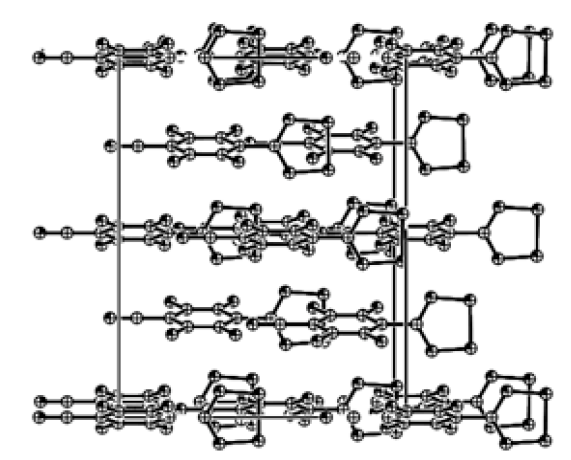

Fig. 14. Molecular packing diagram of the $\square-N C-\mathrm{C}_{6} \mathrm{~F}_{4}-\mathrm{CNSSN} \cdot$ radical viewed perpendicular to the $b$ axis. 


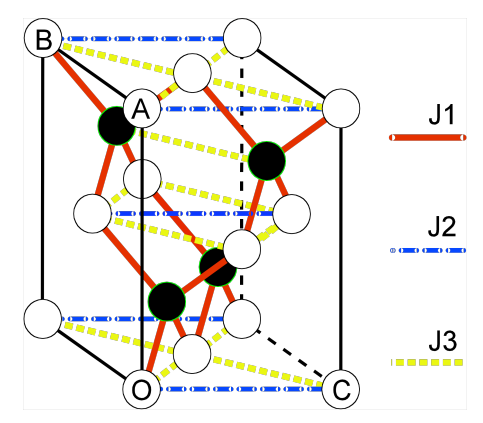

Fig. 15. Scheme of the possible magnetic pathways in the unit cell of the $\square-N C-\mathrm{C}_{6} \mathrm{~F}_{4}-\mathrm{CNSSN}$ • radical. The radicals are represented by spheres. The $J_{4}$ interaction has been omitted for clarity.

The crystal packing viewed perpendicular to the $b$ and $c$ axis is represented in Fig. 14 .

At $36 \mathrm{~K}$ this compound exhibits a transition to an antiferromagnetic phase. Since the two sub-networks with antiparallel spins are not related by a centre of symmetry, the Dzyaloshinskii-Moriya term in the spin Hamiltonian is non-zero and allows canting of the spins from colinearity. In turn this results in a weak spontaneous magnetization $[17,18]$. Extrapolation of the spontaneous magnetisation to $0 \mathrm{~K}$ yields a canting angle of $0.26(2)^{\circ}$ with respect to colinearity. This magnetic phase transition has been characterised by magnetic and heat capacity techniques. The average antiferromagnetic structure has been determined from neutron diffraction techniques [18] and it has been further investigated by $\mu$-SR [68] and single crystal EPR experiments [40, 69]. Furthermore, the pressure dependence of the magnetic ordering of this compound shows that Tc raises up to $72 \mathrm{~K}$ at $16.4 \mathrm{kbar}[66,70]$.

The three possible magnetic exchange pathways obtained from the distance cut-off criteria are represented in Fig. 15 and the calculated exchange interactions presented in Table 4. It has proved possible to probe the magnetic pathway via the closest intermolecular $\mathrm{CN}^{\square} \cdots \mathrm{S}^{\square^{+}}$contact between the cyano group and the dithiadiazolyl ring experimentally and has been found to be negligible [71] in agreement with the calculated exchange interaction. Of the remaining two pathways, only one antiferromagnetic interaction, $\boldsymbol{J}_{1}$, is significant and propagates through the lattice in a diamond-like network. This exchange interaction is associated with a short $\mathrm{S} \cdots \mathrm{N}$ contact of $3.488 \AA$ (see Table 4) between heterocyclic rings. The mean-field approximation based upon the $6-311 \mathrm{G}^{* *}$ magnetic coupling constant $\boldsymbol{J}_{1}$, predicts an antiferromagnetic transition at $45.15 \mathrm{~K}$, in good agreement with the experimental value of $36 \mathrm{~K}$.

Table 5.

Broken Symmetry DFT magnetic coupling constants for $1 \square$.

\begin{tabular}{lllll}
\hline Pathway & Contact & $d(\AA)$ & $\begin{array}{l}J\left(\mathrm{~cm}^{-1}\right) \\
\left(6-31 \mathrm{G}^{* *}\right)\end{array}$ & $\begin{array}{l}J\left(\mathrm{~cm}^{-1}\right) \\
\left(6-311 \mathrm{G}^{* *}\right)\end{array}$ \\
\hline$J_{1}$ & $\mathrm{~S} \cdots \mathrm{N}$ & 3.488 & -32.58 & -31.38 \\
$J_{2}$ & $\mathrm{CN} \cdots \mathrm{S}$ & 2.986 & -0.03 & -0.04 \\
$J_{3}$ & $\mathrm{~F} \cdots \mathrm{S}$ & & -0.006 & -0.007
\end{tabular}

Exchange interactions are based upon the crystal data at $160 \mathrm{~K}$ 


\subsection{Exchange Interactions and Magnetic behaviour of $p-\mathrm{BrC}_{6} \mathrm{~F}_{4} \mathrm{CNSSN} \bullet$}

Like 1] and 3, this radical, 2, also crystallizes in a polar space group, Aba2, [39]. In this radical the difference of electronegativity between the bromine and the sulphur atoms is not enough to force a chain-like motif in the structure as in 10 and 10. Instead, as shown in Fig. 16, the radicals are connected along the crystallographic $c$ axis by short $\mathrm{S} \cdots \mathrm{N}$ contacts of $3.175 \AA$ and along the $b$-axis by $\mathrm{N}$...Br contacts. In addition the rings are -stacked in columns parallel to the crystallographic $a$ axis by means of out-of-plane electrostatic interactions between the sulphur atoms of the heterocyclic rings leading to $\mathrm{S} \cdots \mathrm{N}$ and $\mathrm{S} \cdots \mathrm{S}$ contacts in the range 3.675-3.999 $\AA$ (compared with the sum of van der Waals radii perpendicular to the ring plane $(3.2$ and $4.0 \AA$ for $\mathrm{S} \cdots \mathrm{N}$ and $\mathrm{S} \cdots \mathrm{S}$, respectively).

The magnetic susceptibility of 2 above $60 \mathrm{~K}$ follows a Curie-Weiss law with $\square=-27 \mathrm{~K}$. From this value, either an antiferromagnetic transition at $27 \mathrm{~K}$ (within the mean-field approximation) a broad peak around $27 \mathrm{~K}$ due to short range order interactions would be expected. However, the experimental magnetic susceptibility down to $1.8 \mathrm{~K}$ shows neither an antiferromagnetic transition nor a broad peak but increases continually with decreasing temperature [39]. Amongst the several tentative explanations, the existence of competing ferromagnetic and antiferromagnetic interactions has also been proposed as the possible origin of this magnetic behaviour.

The analysis of the magnetic pathways provides seven possible ones within the distance cut-off criteria. However, only three are significant (Table 5). $\boldsymbol{J}_{1}$ and $\boldsymbol{J}_{2}$ connect molecules along the $a$-axis and alternate in sign. The third exchange pathway, $\boldsymbol{J}_{3}$, connects the radicals in the $c$ direction and is antiferromagnetic.

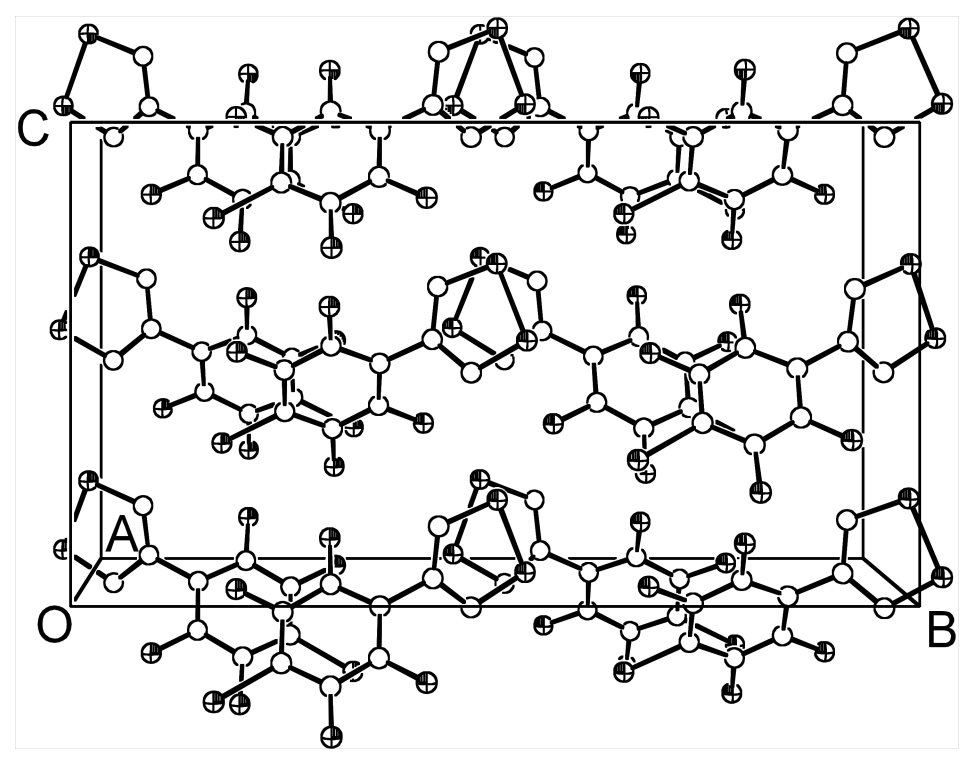

Fig. 16. Molecular packing diagram of $p-B r \mathrm{C}_{6} \mathrm{~F}_{4} \mathrm{CNSSN}$ viewed along the $a$ axis. 


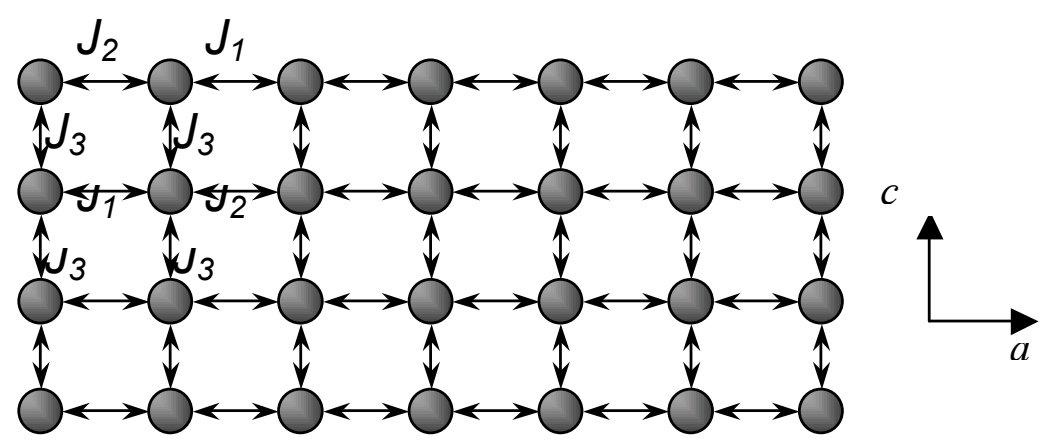

Fig. 17. Exchange pathway in $p-B r \mathrm{C}_{6} \mathrm{~F}_{4} \mathrm{CNSSN}$ with relative orientations of the crystallographic axes

The magnetic motif generated by these three interactions is a two-dimensional grid (Fig. 17) where each molecule simultaneously interacts ferro $\left(\boldsymbol{J}_{2}\right)$ and antiferromagnetically $\left(\boldsymbol{J}_{1}\right.$ and two $\left.\boldsymbol{J}_{3}\right)$. Within the mean-field approximation these interactions lead to an expected Weiss constant of $-26 \mathrm{~K}\left(6-31 \mathrm{G}^{* *}\right)$ or $-24 \mathrm{~K}$ $\left(6-311 \mathrm{G}^{* *}\right)$ in excellent agreement with the experimental value.

The absence of long-range magnetic order can be directly attributed to the combined effects of the very small magnetic anisotropy characteristic of -type orbitals and the two-dimensional nature of the interactions network (Fig. 17). In addition, the competing signs of the exchange interactions leads to frustration in the magnetic system and to the suppression of short-range order.

Table 6.

Broken Symmetry DFT magnetic coupling constants for 2.

\begin{tabular}{ccccc}
\hline Pathway & Contact & $d(\AA)$ & $\begin{array}{c}\boldsymbol{J}\left(\mathrm{cm}^{-1}\right) \\
\left(6-31 \mathrm{G}^{* *}\right)\end{array}$ & $\begin{array}{c}\boldsymbol{J}\left(\mathrm{cm}^{-1}\right) \\
\left(6-311 \mathrm{G}^{* *}\right)\end{array}$ \\
\hline$J_{1}$ & $\mathrm{~N}(1) \ldots \mathrm{S}(1 \mathrm{a})$ & 3.634 & & \\
& $\mathrm{~N}(1) \ldots \mathrm{S}(2 \mathrm{a})$ & 4.182 & & \\
& $\mathrm{~S}(1) \ldots \mathrm{S}(1 \mathrm{a})$ & 3.865 & -9.861 & -7.618 \\
& $\mathrm{~S}(2) \ldots \mathrm{C}(1 \mathrm{a})$ & 4.196 & & \\
$J_{2}$ & $\mathrm{~N}(2) \ldots \mathrm{N}(2 \mathrm{a})$ & 4.377 & & \\
& $\mathrm{~S}(2) \ldots \mathrm{S}(2 \mathrm{a})$ & 3.675 & & +7.519 \\
& $\mathrm{~S}(2) \ldots \mathrm{N}(2 \mathrm{a})$ & 3.738 & +8.513 & \\
$J_{3}$ & $\mathrm{~S}(2) \ldots \mathrm{S}(1 \mathrm{a})$ & 4.371 & & -8.250 \\
\hline
\end{tabular}

Exchange interactions are based upon the crystal data at $160 \mathrm{~K}$.

\section{Other Thiazyl Radicals}

The methodology described in the previous sections, in which magnetic measurements are combined with studies of the spin density distribution in the organic molecule and with $a b$ initio calculations of the exchange pathways, has not been extensively applied to other S-based radicals. There are, however, some interesting 
candidates whose magnetic properties have been investigated although their spin density distribution have not been studied experimentally. Such is the case of some dithiazolyl radicals, such as the methylbenzodithiazolyl, MBDTA, (5), the benzo[1,2:4,5-d']bis[1,3,2]dithiazole, BBDTA, (6) and the trithiatriazapentalenyl, TTTA, (7). Here we extend this approach and show it is equally applicable to other Sbased free radicals. With the exception of TTTA (7) whose properties are described in detail elsewhere in this book [72], no calculations of the strength of the exchange interaction have been reported. We have shown that these calculations are important in determining not only the sign and magnitude of the intermolecular interactions, but also the lattice dimensionality.

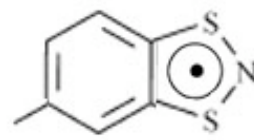

5

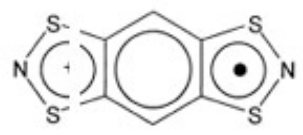

6

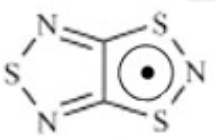

7

\subsection{The Methylbenzodithiazolyl Radical (MBDTA)}

The asymmetric unit of MBDTA (5) contains a single molecule of unexceptional geometry with the fused ring essentially planar. Molecules of MBDTA form a herringbone array with $\square$-stacking along the crystallographic $c$-axis. The distance between equivalent atoms in neighbouring rings in the stack coincides with the length of the $c$-axis, but the molecular plane is inclined at $60.7^{\circ}$ to the $a b$ plane and the closest contact along the stacking direction is $3.742 \AA$ [73]. A view of MBDTA in the $a b$ plane is shown in Fig. 18a. In addition to the intra-stack contacts, there are a series of inter-stack $S \cdots S$ contacts in the range 3.71-3.82 $\AA$. These intermolecular contacts give rise to a two-dimensional sheet of interactions in the crystallographic $b c$ plane.

The magnetic behaviour of (5) exhibits a broad maximum at 140K (Fig. 18b). The absence of any field dependent magnetisation below $140 \mathrm{~K}$ indicates that the maximum in $\square$ is associated with the onset of low dimensional antiferromagnetic order. Below ca. $25 \mathrm{~K}$ the susceptibility curve increases rather sharply most likely due to the contribution from a small mole fraction of non correlated paramagnetic centres in the sample [73].

The low dimensionality of the magnetic structure is consistent with the twodimensional nature of the dithiazolyl interactions in the crystal structure and the compound was analysed as a two dimensional Heisenberg system, using high temperature series expansions for the square planar Heisenberg model [74]. A good agreement between experimental data and this theoretical model was obtained with an exchange term, $\boldsymbol{J} \sim-72 \mathrm{~K}$.

Whilst the fused nature of the BDTA and MBDTA ring systems provides an opportunity for $\square$-delocalisation of the spin density away from the heterocyclic ring, theoretical calculations have shown that the majority of the spin density is still localised on the $\mathrm{S}$ and $\mathrm{N}$ atoms. Consequently the main pathway for magnetic exchange can be considered to be via the intermolecular $\mathrm{S} \cdots \mathrm{N}$ and $\mathrm{S} \cdots \mathrm{S}$ interactions already described. This gives rise to a two-dimensional sheet structure in the $b c$ plane; propagation along 


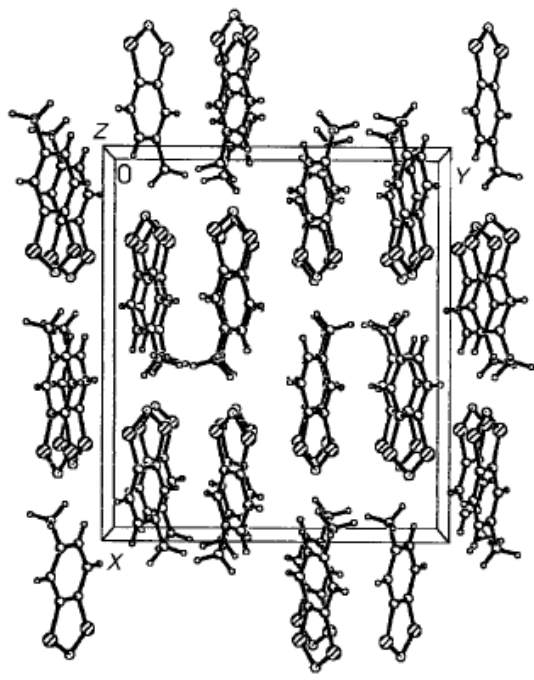

(a)

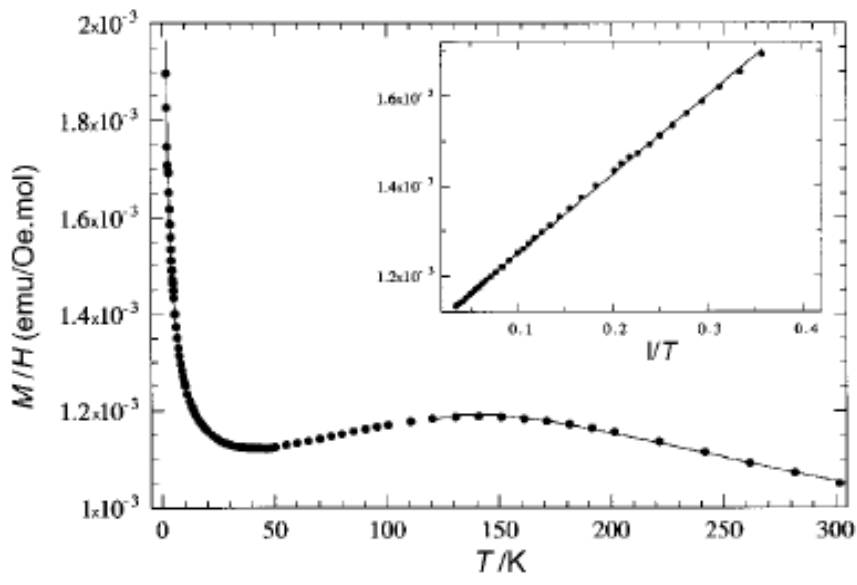

(b)

Fig. 18. a) View of MBDTA in the $a b$ plane; b) Temperature dependence of MBDTA.

the crystallographic $a$ direction relies on $\mathrm{N} \cdots \mathrm{H}$ interactions. A determination of the calculated exchange interactions reveal three dominant interactions propagated via close contacts between heterocyclic rings. The dominant interaction is antiferromagnetic $\left(\boldsymbol{J}_{1}=-92.3 \mathrm{~K}\right.$ at the $6-311 \mathrm{G}^{* *}$ level $)$ and propagates along the crystallographic $c$-axis, whilst the other two are ferromagnetic $\left(\boldsymbol{J}_{2}=+1.9\right.$ and $\left.\boldsymbol{J}_{3}=7.8 \mathrm{~K}\right)$ and propagate along the $b$-axis [54].

\subsection{The benzo[1,2:4,5-d']bis[1,3,2]dithiazole (BBDTA)}

This compound, which preparaton was reported some time ago $[10,75,76]$, is diamagnetic despite the fact that its structure consists in discrete, unassociated molecules [77]. The interest of this compound stays in its radical cation salts $[\mathrm{BBDTA}]\left[\mathrm{Fe}^{\mathrm{III}} \mathrm{Cl}_{4}\right] \cdot \mathrm{CH}_{3} \mathrm{CN},(\mathbf{6 a})$ and [BBDTA] $\left[\mathrm{GaCl}_{4}\right] \cdot \mathrm{CH}_{3} \mathrm{CN}(\mathbf{6 b})$. In these crystals the BBDTA molecules form a ladder-type structure through which the $\left[\mathrm{XCl}_{4}\right]$ anions are weakly linked. Mössbauer experiments in (6a) indicate antiferromagnetic ordering below 6.6K [78]. Instead (6b) is diamagnetic. However, removal of the crystal solvent results in a drastic change from diamagnetic to paramagnetic. The bulk desolvated solid orders as a ferromagnet below $6.7 \mathrm{~K}$ despites the fact that the $\left[\mathrm{GaCl}_{4}\right]$ is a non-magnetic anion [23]. It is certain that the removal of solvent molecules induces a packing modification in the BBDTA radical cations that triggers dominant ferromagnetic interactions. 


\subsection{The Trithiatriazapentalenyl, (TTTA)}

This compound (7), first reported by [79], has been found to exhibit a first-order structural phase transition with a wide thermal hysteresis loop in the vicinity of room temperature that strongly affects its magnetism [80, 81, 82]. Since it is discussed in detail in another chapter of this book [72] only a brief account of its magneto-structural behaviour will be given here.

DFT calculations using the molecular geometry determined from the single crystal crystal studies reported by Wolmershauser [79] indicate that the unpaired electron resides in a $\square^{*}$ orbital which is delocalised over the entire molecule [81]. However, the spin density distribution is asymmetric with $84 \%$ based on the SNS fragment and just $22 \%$ on the NSN fragment. The slight excess of spin density on the heteroatoms, $\mathrm{N}$ and $\mathrm{S}$, is compensated by a small (3\%) negative spin density at $\mathrm{C}$. The high temperature (HT) phase, which structure consists of a regular polar stack of molecules along the $b$-axis, exhibits an effective magnetic moment of $1 \mu_{\mathrm{B}}$ at room temperature, significantly less than that anticipated for a $S=1 / 2$ paramagnet. The magnetic data for this phase $(225-350 \mathrm{~K})$ was modeled as a one-dimensional Heisenberg chain of $\mathrm{S}=1 / 2$ ions and yielded an intra-stack coupling of $\boldsymbol{J}=-320 \mathrm{~K}$, but required an additional inter-stack coupling of $\boldsymbol{J}^{\prime}=-60 \mathrm{~K}[80]$. Although the one-dimensional chain model reproduces the experimental data, the magnitude of $\boldsymbol{J} / \boldsymbol{J}(\sim 0.2)$ indicates that this is not a good onedimensional system. On cooling below $225 \mathrm{~K}$ and by $200 \mathrm{~K}$ the paramagnetism is essentially quenched, consistent with the strong dimerisation along the stacking direction induced by the structural change leading to the low temperature (LT) phase.

On warming the LT phase to room temperature, the susceptibility is essentially constant to $310 \mathrm{~K}$ but then increases abruptly, reaching the susceptibility of the original HT phase at $330 \mathrm{~K}$. The observation that the sample is essentially diamagnetic to $310 \mathrm{~K}$ is consistent with a strongly antiferromagnetically coupled regime. Using the BleaneyBowers equation for an exchange-coupled dimer of $S=1 / 2$ ions [83], the singlet-triplet separation must be in excess of $2 \boldsymbol{J}=-2071 \mathrm{~K}$. This is in agreement with the value reported by Awaga $(\boldsymbol{J}=-1300 \mathrm{~K})$ [80]. DFT calculations on the LT, dimeric, phase indicated that the ground state electronic configuration is an open shell singlet with a singlet-triplet separation of $2 \boldsymbol{J}=-2657 \mathrm{~K}$. This is in contrast to many dithiadiazolyl radicals [84] and other dithiazolyl radicals $[85,86]$ in which a closed-shell singlet (spinpaired dimer) is the electronic ground state.

A key question inherent to TTTA is its bistability. In order to achieve bistability, there must be two polymorphs of comparable energy. Polymorphism is not uncommon, particularly in molecular materials in which the forces between molecules are often weak, and a number of thiazyl-based radicals have been shown to exhibit polymorphism $[87,88,89]$ but do not exhibit bistability. The bistability arises because the energy pathway to interconversion must not be too prohibitive. In the case of 7 the differences in intra-stack exchange energy favour the LT phase whereas electrostatic interactions seem to favour the HT phase [81]. 


\section{Conclusions}

This chapter has overviewed a series of S-based molecular magnetic materials of interest because of their magnetic ordering and magnetic interactions. It emphasises the importance of the spin density distribution determinations and ab initio calculations of the exchange interactions to complement the information gained from magnetic measurements. This approach allows a deep understanding of the interaction mechanisms governing the magnetism of this type of compounds and the way interactions propagate.

It has been shown that in the case of the dithiadiazolyl radicals the spin density is almost entirely located on the sulphur and nitrogen atoms of the dithiadiazolyl ring reflecting the nature of the SOMO orbital. The magnetism of four compounds of this series presenting very different magnetic behaviour, ferromagnetism, antiferromagnetism, paramagnetism and spin frustration have been discussed.

The presence of ferromagnetic interactions in three of the five radical structures indicates that dithiadiazolyl rings are good candidates in the quest to synthesize purely organic magnets with higher transition temperatures. An adequate molecular design could lead to suitable crystal packing with relatively strong ferromagnetic interactions propagating in the three dimensions. The magnitude of the ferromagnetic exchange couplings determined here would give an upper limit to ferromagnetic ordering temperatures of ca. $10^{1} \mathrm{~K}$. Conversely the antiferromagnetic interactions are substantially stronger and could lead to ordering temperatures up to $10^{2} \mathrm{~K}$. Approaches to generate high $\mathrm{T}_{\mathrm{C}}$ magnets might therefore be to focus on strategies which favour weak ferromagnetism, as in the case of 1】, or develop novel two-spin systems to form ferromagnetic materials.

It would be of interest to extend these studies to other series of S-based radicals, in particular to the dithiazolyl-based compounds which magnetism seems to be as rich as in the case of the dithiadiazoly ones.

\section{References}

1. M. Tamura, Y. Nakazawa, D. Shiomi, K. Nozawa, Y. Hosokoshi, M. Ishikawa, M. Takahashi, and M. Kinoshita, Chem. Phys. Lett. 186 (1991) 401.

2. P.M. Allemand, K.C. Khemani, A. Koch, F. Wudl, K. Holczer, S. Donovan, G. Gruner, and J.D. Thompson, Science 253 (1991) 301.

3. J. Veciana, in: Molecular Magnetism: From Molecular Assemblies to the Devices, Eds. E. Coronado, P. Delhaes, D. Gatteschi, and J. S. Miller, NATO ASI series, 321 (1996) 425, Kluwer Acad. Publ.

4. V. Gadet, T. Mallah, I. Castro, P. Veillet, and M. Verdaguer, J. Am. Chem. Soc. 114 (1992) 9213.

5. M. Deumal, J.J. Novoa, M.J. Bearpark, P. Celani, M. Olivucci, and M.A. Robb, J. Phys. Chem. A 102 (1998) 8404.

6. J.J. Novoa and M. Deumal, Struct. and Bonding 100 (2001) 33.

7. H. M. McConnell, Proc. Robert A. Welch Found. Conf. Chem. Res. 11 (1967) 144.

8. J. Thomaides, P. Maslak, and R. Breslow, J. Am Chem. Soc. 110 (1988) 3970.

9. J.B. Torrance, I. Bagus, A.I. Nazzal, and S.P. Parkin, J. Appl. Phys. 63 (1988) 2962. 
10. E. Dorman, M.J. Nowak, K. Williams, R.G. Angus, and F.J. Wudl, J. Am. Chem. Soc. 109 (1987) 2594.

11. J.S. Miller, J.C. Calabrese, H. Rommelmann, S.R. Chittapedi, R.W. Zhang, W.M. Reiff, and A.J. Epstein, J. Am. Chem. Soc. 109 (1987) 769.

12. J. Schweizer, S. Golhen, E. Lelievre-Berna, L. Ouahab, Y. Pontillon, and E. Ressouche, Physica B 297 (2001) 213.

13. C. Herring, Magnetism IIB, Direct exchange between well-separated atoms, 5, Eds. G.T. Rado and H. Suhl (1966) Academic Press, New York.

14. I. Dzyaloshinsky, J. Phys. Chem. Solids 4 (1958) 241.

15. T. Moriya, Phys. Rev. 120 (1960) 91.

16. R.K. Kremer, B. Kanellakopulos, P. Bele, H. Brunner, and F.A. Neugebauer, Chem. Phys. Lett. 230 (1994) 255.

17. A.J. Banister, N. Bricklebank, I. Lavender, J.M. Rawson, C.I. Gregory, B.K. Tanner, W. Clegg, M.R.J. Elsegood, and F. Palacio, Angew. Chem. Int. Ed. Engl. 35 (1996) 21, 2533.

18. F. Palacio, G. Antorrena, M. Castro, R. Burriel, J.M. Rawson, J.N.B. Smith, N. Bricklebank, J.J. Novoa, and C. Ritter, Phys. Rev. Lett. 79 (1997) 2336.

19. M. Kinoshita, 2001, $\square$ Electron Magnetism: from Molecules to Magnetic Materials, Ed. J. Veciana, Springer Verlag, Berlin, p. 1

20. P.M. Allemand, K. C. Khemani, A. Koch, F. Wudl, K. Holczer, S. Donovan, G. Gruner and J. D. Thompson, Science 253 (1991) 301.

21. R. Chiarelli, M.A. Novak, A. Rassat, and J. L. Tholence, Nature 363 (1993) 147.

22. A. Alberola, R.J. Less, C.M. Pask, J.M. Rawson, F. Palacio, P. Oliete, C. Paulsen, A. Yamaguchi, and R. D. Farley, Angew. Chem. Int. Ed. Engl. 42 (2003) 4782.

23. W. Fujita and K. Awaga, Chem. Phys. Lett. 357 (2002) 385.

24. P.M. Lahti, Magnetic Properties of Organic Materials, Editor, (1999) Marcel Dekker Inc. (NY).

25. G. Antorrena, J.E. Davies, M. Hartley, F. Palacio, J.M. Rawson, J.N. B. Smith, and A. Steiner, Chem. Comm. 15 (1999) 1393.

26. F. Palacio and J.M. Rawson, in: Magnetic Properties of Organic Materials. Ed. by P.M. Lahti (1999) 703, Marcel Dekker Inc. (NY).

27. J.M. Rawson and G.D. McManus, Coord. Chem. Rev. 189 (1999) 135.

28. N. Burford, J. Passmore, and M.J. Schriver, J. Chem. Soc, Chem. Commun. 140 (1986).

29. A.W. Cordes, R.C. Haddon, and R.T. Oakley, The Chemistry of Inorganic Ring Systems, 295 (1992) Eds. Steudel, Elsevier, Amsterdam, The Netherlands.

30. J.M. Rawson and A.J. Banister, The Chemistry of Inorganic Ring Systems, Eds. R. Steudel, 323 (1992) Elsevier, Amsterdam, The Netherlands.

31. J.M. Rawson, A.J. Banister, and I. Lavender, Adv. Heterocycl. Chem. 62 (1995) 137.

32. J. Campbell, D. Klapstein, P.F. Bernath, W.M. Davis, R.T. Oakley, and J.D. Goddard, Inorg. Chem. 35 (1996) 4264.

33. J.M. Rawson and F. Palacio, $\square$ Electron Magnetism: from Molecules to Magnetic Materials, Ed. J. Veciana, (2001) 93, Springer Verlag, Berlin.

34. J.M. Rawson, R.J. Lessand J.N.B. Smith, F. Palacio, and G. Antorrena, Mol. Cryst. Liq. Cryst. 334 (1999) 275.

35. A.J. Banister, N. Bricklebank, W. Clegg, M.R.J. Elsegood, C.I. Gregory, I. Lavender, J.M. Rawson, and B.K. Tanner, J. Chem. Soc. Chem. Comm. 6 (1995) 679.

36. G. Antorrena, F. Palacio, J.M. Rawson, and J.N.B. Smith, in: Supramolecular Engineering in Synthetic Metallic Materials. Ed. by J. Veciana, C. Rovira, and D.B. Amabilino, NATO ASI, C518 (1999) 217, Kluwer Publ.

37. C.M. Pask, R.J. Less, J.M. Rawson, F. Palacio, and P. Oliete, Phosphorus Sulfur. 168 (2001) 457. 
38. A. Alberola, R.J.Less, F. Palacio, C.M. Pask and J.M. Rawson, Molecules, 9 (2004) 771.

39. G. Antorrena, J. E. Davies, M. Hartley, F. Palacio, J. M. Rawson, J. N. B. Smith and A. Steiner, Chem. Comm., 15 (1999) 1393.

40. A. Alberola, C. M. Pask, J. M. Rawson, E.J.L. McInnes, J. Wolowska, H. El-Mkami and G.M. Smith, J. Phys. Chem. B, 107 (2003) 14158.

41. C.D. Bryan C.D., A.W. Cordes, R.M. Fleming, N.A. George, S.H. Glarum, R.C. Haddon, C.D. MacKinnon, R.T. Oakley, T.T.M. Palstra and A.S. Perel, J. Am. Chem. Soc., 117 (1995) 6880.

42. R.A. Beekman, R.T. Boere, K.H. Moock, and M. Parvez, Can J. Chem., 76 (1998) 85.

43. T.M. Barclay, A.W. Cordes, N.A. George, R.T. Oakley, R.C. Haddon, and M.E. Itkis, Chem. Commun. (1999) 2269.

44. G. Antorrena, Estudio de Materiales Magnéticos Moleculares: Radicales S-N Y Polímeros de Coordinación., (1998) Thesis, University of Zaragoza, Zaragoza.

45. P.J. Alonso, G. Antorrena, J.I. Martinez, J.J. Novoa, F. Palacio, J.M. Rawson, and J.N.B. Smith, Appl. Magn. Reson. 20 (2001) 231.

46. P.J. Brown, A. Capiomont, B. Gillon, and J. Schweizer, J. Magn. Magn. Mater. 14 (1979) 289.

47. P.J. Brown, A. Capiomont, B. Gillon, and J. Schweizer, Mol. Phys. 48 (1983) 4.

48. A. Zheludev, V. Barone, M. Bonnet, B. Delley, A. Grand, E. Ressouche, P. Rey, R. Subra, and J. Schweizer, J. Am. Chem. Soc. 116 (1994) 2019.

49. J. Luzón, J. Campo, F. Palacio, G.J. McIntyre, J.M. Rawson, A. Alberola, and A. Goeta, 2005, (submitted to publication).

50. W.J. Hehre, R.F. Stewart, and J.A. Pople, J. Chem. Phys. 51 (1969) 2657.

51. W.J. Hehre, R. Ditchfield, R.F. Stewart, and J.A. Pople, J. Chem. Phys. 52 (1970) 2769.

52. E. Ressouche, Densité de Spin Dans Les Radicaux Libres Nitroxides et Leurs Complexes Cuivriques, Thesis of Université Joseph Fourier (1991) Grenoble.

53. Gaussian 98, Revision A.11.3, M.J. Frisch, G.W. Trucks, H.B. Schlegel, G.E. Scuseria, M.A. Robb, J.R. Cheeseman, V.G. Zakrzewski, J.A. Montgomery, Jr. , R.E. Stratmann, J.C. Burant, S. Dapprich, J.M. Millam, A.D. Daniels, K.N. Kudin, M.C. Strain, O. Farkas, J. Tomasi, V. Barone, M. Cossi, R. Cammi, B. Mennucci, C. Pomelli, C. Adamo, S. Clifford, J. Ochterski, G.A. Petersson, P.Y. Ayala, Q. Cui, K. Morokuma, N. Rega, P. Salvador, J.J. Dannenberg, D.K. Malick, A.D. Rabuck, K. Raghavachari, J.B. Foresman, J. Cioslowski, J.V. Ortiz, A.G. Baboul, B.B. Stefanov, G. Liu, A. Liashenko, P. Piskorz, I. Komaromi, R. Gomperts, R.L. Martin, D.J. Fox, T. Keith, M.A. Al-Laham, C.Y. Peng, A. Nanayakkara, M. Challacombe, P.M.W. Gill, B. Johnson, W. Chen, M.W. Wong, J.L. Andres, C. Gonzalez, M. Head-Gordon, E.S. Replogle, and J.A. Pople, Gaussian, Inc. , Pittsburgh PA, (2002).

54. J. Luzón, Magnetic Interaction Mechanisms in Molecular Materials, (2004) Thesis, University of Zaragoza, Zaragoza.

55. J.M. Rawson, J. Luzón, and F. Palacio, Coord. Chem. Rev. (2005) in press.

56. M. Deumal, J. Ribas-Arino, M.A. Robb, J. Ribas, and J.J. Novoa, Molecules 9 (2004) 757.

57. L. Noodleman and J.G. Norman, J. Chem. Phys. 70 (1979) 4903.

58. L. Noodleman, J. Chem. Phys. 74 (1981) 5737.

59. L. Noodleman and E.R. Davidson, Chem. Phys. 109 (1985) 131.

60. H. Nagao, M. Nishino, Y. Shigeta, T. Soda, Y. Kitagawa, T. Onishi, Y. Yoshioka, and K. Yamaguchi, Coord. Chem. Rev. 198 (2000) 265.

61. E. Ruiz, J. Cano, S. Alvarez and P. Alemany, J. Chem. Comp. 20 (1999) 1391.

62. R. Krishnan, J.S. Binkley, R. Seeger, and J.A. Pople, J. Chem. Phys. 72 (1980) 650. 
63. C. Herring, Magnetism IIB, Direct exchange between well-separated atoms, 5, Eds. G.T. Rado and H. Suhl (1966) Academic Press, New York.

64. M. Deumal and J.J. Novoa, J. Mol. Struc. 506 (2000) 287.

65. M. Deumal, J.J. Novoa, M.J. Bearpark, P. Celani, M. Olivucci, and M.A. Robb, J. Phys. Chem. A 106 (2001) 1299.

66. K. Takeda and M. Mito, in: Carbon-based magnetism. Ed. by T. Makarova and F. Palacio. Elsevier. p 139.

67. K. Takeda, Y. Yoshida, Y. Inagaki, T. Kawae, J.M. Rawson, F. Palacio, and M. Mito, in preparation for publication.

68. F.L. Pratt, A.E. Goeta, F. Palacio, J.M. Rawson, and J.N.B. Smith, Physica B289 (2000) 119.

69. J.M. Rawson, A. Alberola, H. El-Mkami, and G.M. Smith, J. Phys. Chem. Sol. 65 (2004) 727.

70. M. Mito, T. Kawae, K. Takeda, S. Takagi, Y. Matsushita, H. Deguchi, J. M. Rawson, and F. Palacio, Polyhedron 20 (2001) 1509.

71. P.J. Langley, J.M. Rawson, J.N.B. Smith, M. Schuler, R. Bachmann, A. Schweiger, F. Palacio, G. Antorrena, G. Gescheidt, and A. Quintel, J. Mater. Chem. 9 (1999) 1431.

72. K. Awaga, W. Fujita, T. Tanaka, H. Matsuzaki, and H. Okamoto, in: Carbon-based magnetism. Ed. by Makarova, T. and F. Palacio. Elsevier (2006). p. 189.

73. G.D. McManus, J.M. Rawson, N. Feeder, F. Palacio, and P. Oliete, J. Mater. Chem. 10 (2000) 2001.

74. R. Navarro, in: Magnetic Properties of Layered Transition Metal Compounds. Ed. L.J. de Jongh. (1990) Kluwer Acad.

75. G. Wolmershäuser, M. Schnauber, T. Wilhelm, and L.H.Sutcliffe, Synth. Met. 14 (1986) 239.

76. K.A. Williams, M.J. Nowak, E. Dormann, and F. Wudl, Synth. Met. 14 (1986) 233.

77. T.M. Barclay, A.W. Cordes, R.H. de Laat, J.D. Goddard, R.C. Haddon, D.Y. Jeter, R.C. Mawhinney, R.T. Oakley, T.T.M. Palstra, G.W. Patenaude, R.W. Reed, and N.P.C. Westwood, J. Am. Chem. Soc. 119 (1997) 2633.

78. G. Wolmershäuser, G. Wortman, and M. Schnauber, J. Chem. Research (S) (1988) 358.

79. G. Wolmershäuser and R. Johann, Angew. Chem., Int. Ed., Engl. 28 (1989) 920.

80. W. Fujita and K. Awaga, Science 286 (1999) 261.

81. G.D. McManus, J.M. Rawson, N. Feeder, J. van Duijn, E.J.L McInnes, J.J. Novoa, R. Burriel, F. Palacio, and P. Oliete, J. Mater. Chem. 11 (2001) 1992.

82. W. Fujita, K. Awaga, H. Matsuzaki, and H. Okamoto, Phys. Rev. B65 (2002) 064434.

83. B. Bleaney and K.D. Bowers, Proc. Roy. Soc. (London) Ser. A. 214 (1952) 451.

84. A.W. Cordes, C.D. Bryan, W.M. Davis, R.H. de Laat, S.H. Glarum, J.D. Goddard, R.C. Haddon, R.G. Hicks, D.K. Kennepohl, R.T. Oakley, S.R. Scott, and N.P.C. Westwood, J. Am. Chem. Soc. 115 (1993) 7232.

85. E.G. Awere, N. Burford, R.C. Haddon, S. Parsons, J. Passmore, J.V. Waszczak, and P.S. White, Inorg. Chem. 29 (1990) 4821.

86. E.G. Awere, N. Burford, C. Mailer, J. Passmore, M.J. Schriver, P.S. White, A.J. Banister, H. Oberhammer, and L.H. Sutcliffe, J. Chem. Soc. Chem. Commun. (1987) 66.

87. A.W. Cordes, R.C. Haddon, R.G. Hicks, R.T. Oakley, and T.T.M. Palstra, Inorg. Chem. 31 (1992) 1802.

88. A.W. Cordes, R.C. Haddon, R.G. Hicks, R.T. Oakley, T.T.M. Palstra, L.F. Schneemeyer, and J.V. Waszczak, J. Am. Chem. Soc. 114 (1992) 1729.

89. C.D. Bryan, A.W. Cordes, R.C. Haddon, R.G. Hicks, D.K. Kennepohl, C.D. McKinnon, R.T. Oakley, T.T.M. Palstra, A.S. Perel, S.R. Scott, L.F. Schneemeyer, and J.V. Waszczak, J. Am. Chem. Soc. 116 (1994) 1205. 